TRANSACTIONS OF THE

AMERICAN MATHEMATICAL SOCIETY

Volume 359, Number 10, October 2007, Pages 4977-4997

S 0002-9947(07)04211-0

Article electronically published on May 7, 2007

\title{
THE VECTORIAL RIBAUCOUR TRANSFORMATION FOR SUBMANIFOLDS AND APPLICATIONS
}

\author{
M. DAJCZER, L. A. FLORIT, AND R. TOJEIRO
}

\begin{abstract}
In this paper we develop the vectorial Ribaucour transformation for Euclidean submanifolds. We prove a general decomposition theorem showing that under appropriate conditions the composition of two or more vectorial Ribaucour transformations is again a vectorial Ribaucour transformation. An immediate consequence of this result is the classical permutability of Ribaucour transformations. Our main application is to provide an explicit local construction of an arbitrary Euclidean $n$-dimensional submanifold with flat normal bundle and codimension $m$ by means of a commuting family of $m$ Hessian matrices on an open subset of Euclidean space $\mathbb{R}^{n}$. Actually, this is a particular case of a more general result. Namely, we obtain a similar local construction of all Euclidean submanifolds carrying a parallel flat normal subbundle, in particular of all those that carry a parallel normal vector field. Finally, we describe all submanifolds carrying a Dupin principal curvature normal vector field with integrable conullity, a concept that has proven to be crucial in the study of reducibility of Dupin submanifolds.
\end{abstract}

An explicit construction of all submanifolds with flat normal bundle of the Euclidean sphere carrying a holonomic net of curvature lines, that is, admitting principal coordinate systems, was given by Ferapontov in [8]. The author points out that his construction "resembles" the vectorial Ribaucour transformation for orthogonal systems developed in [13. The latter provides a convenient framework for understanding the permutability properties of the classical Ribaucour transformation.

This paper grew from an attempt to better understand the connection between those two subjects, as a means of unravelling the geometry behind Ferapontov's construction. This has led us to develop a vectorial Ribaucour transformation for Euclidean submanifolds, extending the transformation in [13] for orthogonal coordinate systems. It turns out that any $n$-dimensional submanifold with flat normal bundle of $\mathbb{R}^{n+m}$ can be locally transformed by a suitable vectorial Ribaucour transformation to the inclusion map of an open subset of an $n$-dimensional subspace of $\mathbb{R}^{n+m}$. Inverting such a transformation yields the following explicit local construction of an arbitrary $n$-dimensional submanifold with flat normal bundle of $\mathbb{R}^{n+m}$ by means of a commuting family of $m$ Hessian matrices on an open subset of $\mathbb{R}^{n}$. Notice that carrying a principal coordinate system is not required.

Theorem 1. Let $\varphi_{1}, \ldots, \varphi_{m}$ be smooth real functions on an open simply connected subset $U \subset \mathbb{R}^{n}$ satisfying

$$
\left[\operatorname{Hess} \varphi_{i}, \operatorname{Hess} \varphi_{j}\right]=0, \quad 1 \leq i, j \leq m,
$$

Received by the editors August 3, 2005.

2000 Mathematics Subject Classification. Primary 53B25, 58J72.

(C)2007 American Mathematical Society 
and let $\mathcal{G}: U \rightarrow M_{n \times m}(\mathbb{R})$ be defined by $\mathcal{G}=\left(\nabla \varphi_{1}, \ldots, \nabla \varphi_{m}\right)$. Then for any $x \in U$ there exists a smooth map $\Omega: V \rightarrow G l\left(\mathbb{R}^{m}\right)$ on an open subset $V \subset U$ containing $x$ such that $d \Omega=\mathcal{G}^{t} d \mathcal{G}$ and $\Omega+\Omega^{t}=\mathcal{G}^{t} \mathcal{G}+I$. Moreover, the map

$$
f(u)=\left(\begin{array}{c}
u^{t}+\mathcal{G} \Omega^{-1} \varphi(u) \\
\Omega^{-1} \varphi(u)
\end{array}\right)
$$

with $u=\left(u_{1}, \ldots, u_{n}\right) \in V$ and $\varphi(u)=\left(\varphi_{1}(u), \ldots, \varphi_{m}(u)\right)^{t}$ defines, at regular points, an immersion $f: V \rightarrow \mathbb{R}^{n+m}$ with flat normal bundle.

Conversely, any isometric immersion $f: M^{n} \rightarrow \mathbb{R}^{n+m}$ with flat normal bundle can be locally constructed in this way.

The case of submanifolds of the sphere can be easily derived from the preceding result and the observation that any such submanifold arises as the image of a unit parallel normal vector field to a submanifold with flat normal bundle of Euclidean space (see Corollary 19). In this way we recover Ferapontov's result for the holonomic case (see Theorem 201), thus proving his guess correct.

Theorem 1 is actually a particular case of a more general result. In fact, we obtain a similar local explicit construction (see Theorem 18) of all isometric immersions $\tilde{f}: \tilde{M}^{n+m} \rightarrow \mathbb{R}^{n+m+p}$ carrying a parallel flat normal subbundle of rank $m$, in particular of all those that carry a parallel normal vector field, starting with an isometric immersion $f: M^{n} \rightarrow \mathbb{R}^{n+p}$ and a set of Codazzi tensors $\Phi_{1}, \ldots, \Phi_{m}$ on $M^{n}$ that commute one with each other and with the second fundamental form of $f$. We refer the reader to [1] for results of a global nature on such isometric immersions, with strong implications for the submanifold geometry of orbits of orthogonal representations.

By putting together the preceding result with Theorem 8 of [6], we obtain an explicit construction (see Theorem 22) in terms of the vectorial Ribaucour transformation of all Euclidean submanifolds that carry a Dupin principal curvature normal vector field with integrable conullity (see Section 7 for the precise definitions), a concept that has proven to be crucial in the study of reducibility of Dupin submanifolds (see [6]).

A key feature of the Ribaucour transformation for submanifolds (in particular, orthogonal systems) is its permutability property. Namely, given two Ribaucour transforms of a submanifold, there is, generically, a fourth submanifold that is a simultaneous Ribaucour transform of the first two, giving rise to a Bianchi quadrilateral.

More generally, for any integer $k \geq 2$ we define a Bianchi $k$-cube as a $(k+1)$-tuple $\left(\mathcal{C}_{0}, \ldots, \mathcal{C}_{k}\right)$, where each $\mathcal{C}_{i}, 0 \leq i \leq k$, is a family of submanifolds with exactly $\left(\begin{array}{l}k \\ i\end{array}\right)$ elements, such that every element of $\mathcal{C}_{1}$ is a Ribaucour transform of the unique element of $\mathcal{C}_{0}$ and such that, for every $\hat{f} \in \mathcal{C}_{s+1}, 1 \leq s \leq k-1$, there exist unique elements $\hat{f}_{1}, \ldots, \hat{f}_{s+1} \in \mathcal{C}_{s}$ satisfying the following conditions:

(i) $\hat{f}$ is a Ribaucour transform of $\hat{f}_{1}, \ldots, \hat{f}_{s+1}$.

(ii) For each pair of indices $1 \leq i \neq j \leq s+1$ there exists a unique element $\hat{f}_{i j} \in \mathcal{C}_{s-1}$ such that $\left\{\hat{f}_{i j}, \hat{f}_{i}, \hat{f}_{j}, \hat{f}\right\}$ is a Bianchi quadrilateral.

The following Bianchi $k$-cube theorem was proved in [11] for $k=3$ in the context of triply orthogonal systems of Euclidean space. A nice proof in the setup of Lie sphere geometry was recently given in [2], where an indication was also provided of how the general case can be settled by using results of [12] for discrete orthogonal nets together with an induction argument. 


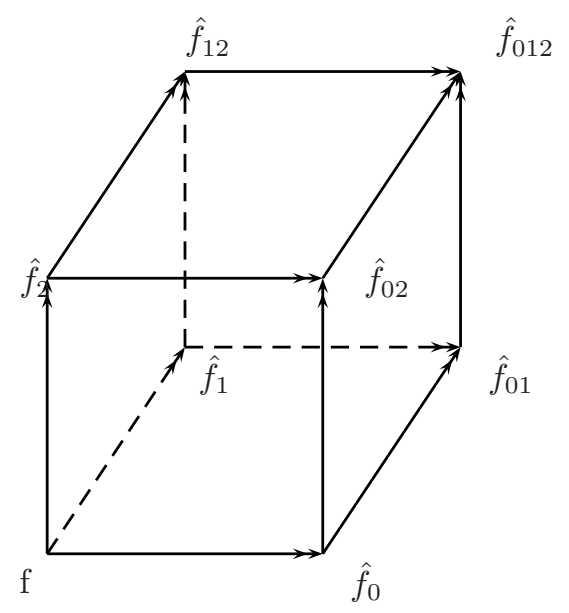

Theorem 2. Let $f: M^{n} \rightarrow \mathbb{R}^{N}$ be an isometric immersion and let $f_{1}, \ldots, f_{k}$ be independent Ribaucour transforms of $f$. Then, for a generic choice of simultaneous Ribaucour transforms $f_{i j}$ of $f_{i}$ and $f_{j}$ such that $\left\{f_{i j}, f_{i}, f_{j}, f\right\}$ is a Bianchi quadrilateral for all pairs $\{i, j\} \subset\{1, \ldots, k\}$ with $i \neq j$, there exists a unique Bianchi $k$-cube $\left(\mathcal{C}_{0}, \ldots, \mathcal{C}_{k}\right)$ such that $\mathcal{C}_{0}=\{f\}, \mathcal{C}_{1}=\left\{f_{1}, \ldots, f_{k}\right\}$ and $\mathcal{C}_{2}=\left\{f_{i j}\right\}_{1 \leq i \neq j \leq k}$.

We give a simple and direct proof of Theorem 2 in Section 5, where the precise meanings of independent and generic are explained. The proof relies on a general decomposition theorem for the vectorial Ribaucour transformation for submanifolds (Theorem 14), according to which the composition of two or more vectorial Ribaucour transformations with appropriate conditions is again a vectorial Ribaucour transformation. The latter extends a similar result of 13 for the case of orthogonal systems and implies, in particular, the classical permutability of Ribaucour transformations for surfaces and, more generally, the permutability of vectorial Ribaucour transformations for submanifolds.

\section{Preliminaries}

Let $M^{n}$ be an $n$-dimensional Riemannian manifold and let $\xi$ be a Riemannian vector bundle over $M^{n}$ endowed with a compatible connection $\nabla^{\xi}$. We denote by $\Gamma(\xi)$ the space of smooth sections of $\xi$ and by $R^{\xi}$ its curvature tensor. If $\zeta=\xi^{*} \otimes \eta=\operatorname{Hom}(\xi, \eta)$ is the tensor product of the vector bundles $\xi^{*}$ and $\eta$, where $\xi^{*}$ stands for the dual vector bundle of $\xi$ and $\eta$ is a Riemannian vector bundle over $M^{n}$, then the covariant derivative $\nabla Z \in \Gamma\left(T^{*} M \otimes \zeta\right)$ of $Z \in \Gamma(\zeta)$ is given by

$$
\left(\nabla_{X}^{\zeta} Z\right)(v)=\nabla_{X}^{\eta} Z(v)-Z\left(\nabla_{X}^{\xi} v\right)
$$

for any $X \in \Gamma(T M)$ and $v \in \Gamma(\xi)$. In particular, if $\omega \in \Gamma\left(T^{*} M \otimes \xi\right)$ is a smooth one-form on $M^{n}$ with values in $\xi$, then $\nabla \omega \in \Gamma\left(T^{*} M \otimes T^{*} M \otimes \xi\right)$ is given by

$$
\nabla \omega(X, Y):=\left(\nabla_{X}^{T^{*} M \otimes \xi} \omega\right)(Y)=\nabla_{X}^{\xi} \omega(Y)-\omega\left(\nabla_{X} Y\right)
$$

where in the right hand side $\nabla$ denotes the Levi-Civita connection of $M^{n}$. The exterior derivative $d \omega \in \Gamma\left(\Lambda^{2} T^{*} M \otimes \xi\right)$ of $\omega$ is related to $\nabla \omega$ by

$$
d \omega(X, Y)=\nabla \omega(X, Y)-\nabla \omega(Y, X)
$$


The one-form $\omega$ is closed if $d \omega=0$. If $Z \in \Gamma(\xi)$, then $\nabla Z=d Z \in \Gamma\left(T^{*} M \otimes \xi\right)$ is the one-form given by $\nabla Z(X)=\nabla_{X}^{\xi} Z$. In case $\xi=M \times V$ is a trivial vector bundle over $M^{n}$, with $V$ a Euclidean vector space, that is, a vector space endowed with an inner product, then $\Gamma\left(T^{*} M \otimes \xi\right)$ is identified with the space of smooth one-forms with values in $V$. We use the same notation for the vector space $V$ and the trivial vector bundle $\xi=M \times V$ over $M^{n}$.

Given $Z_{1} \in \Gamma\left(\xi^{*} \otimes \eta\right)$ and $Z_{2} \in \Gamma\left(\eta^{*} \otimes \gamma\right)$, we define $Z_{2} Z_{1} \in \Gamma\left(\xi^{*} \otimes \gamma\right)$ by

$$
Z_{2} Z_{1}(v)=Z_{2}\left(Z_{1}(v)\right), \quad v \in \Gamma(\xi) .
$$

For $Z \in \Gamma\left(\xi^{*} \otimes \eta\right)$, we define $Z^{t} \in \Gamma\left(\eta^{*} \otimes \xi\right)$ by

$$
\left\langle Z^{t}(u), v\right\rangle=\langle u, Z(v)\rangle, u \in \Gamma(\eta) \text { and } v \in \Gamma(\xi) .
$$

For later use, we summarize in the following lemma a few elementary properties of covariant and exterior derivatives, which follow by straightforward computations.

Lemma 3. The following facts hold:

(i) If $Z_{1} \in \Gamma\left(\xi^{*} \otimes \eta\right)$ and $Z_{2} \in \Gamma\left(\eta^{*} \otimes \gamma\right)$, then $d\left(Z_{2} Z_{1}\right)=\left(d Z_{2}\right) Z_{1}+Z_{2}\left(d Z_{1}\right)$.

(ii) If $Z \in \Gamma\left(\xi^{*} \otimes \eta\right)$, then $d Z^{t}=(d Z)^{t}$.

(iii) If $Z \in \Gamma(\xi)$, then $d^{2} Z(X, Y)=R^{\xi}(X, Y) Z$.

(iv) If $\zeta=\xi^{*} \otimes \eta$ and $Z \in \Gamma(\zeta)$, then

$$
\left(R^{\zeta}(X, Y) Z\right)(v)=R^{\eta}(X, Y) Z(v)-Z\left(R^{\xi}(X, Y) v\right)
$$

We also need the following result.

Proposition 4. Let $\xi, \eta$ be Riemannian vector bundles over $M^{n}$ and

$$
\omega \in \Gamma\left(T^{*} M \otimes \xi\right) .
$$

Set $\zeta=\eta^{*} \otimes T M$ and $\gamma=\eta^{*} \otimes \xi$. Let $\Phi \in \Gamma\left(T^{*} M \otimes \zeta\right)$ be a closed one-form such that

$$
\nabla \omega\left(X, \Phi_{u} Y\right)=\nabla \omega\left(Y, \Phi_{u} X\right) \text { for all } u \in \Gamma(\eta),
$$

where we write $\Phi_{u} X=\Phi(X)(u)$. Then the one-form $\rho=\rho(\omega, \Phi) \in \Gamma\left(T^{*} M \otimes \gamma\right)$ defined by $\rho(X)(u)=\omega\left(\Phi_{u} X\right)$ is also closed.

Proof. We have

$$
\begin{aligned}
\nabla \rho(X, Y)(u) & =\nabla_{X}^{\xi} \rho(Y)(u)-\rho(Y)\left(\nabla_{X}^{\eta} u\right)-\rho\left(\nabla_{X} Y\right)(u) \\
& =\nabla_{X}^{\xi} \omega\left(\Phi_{u} Y\right)-\omega\left(\Phi_{\nabla_{X}^{\eta} u} Y\right)-\omega\left(\Phi_{u} \nabla_{X} Y\right) \\
& =\nabla \omega\left(X, \Phi_{u} Y\right)+\omega(\nabla \Phi(X, Y) u) .
\end{aligned}
$$

The following consequence of Proposition 4 will be used throughout the paper.

Corollary 5. Under the assumptions of Proposition 4, assume further that $M^{n}$ is simply-connected and that $\xi$ and $\eta$ are flat. Then there exists $\Omega(\omega, \Phi) \in \Gamma\left(\eta^{*} \otimes \xi\right)$ such that

$$
d \Omega(\omega, \Phi)(X)(u)=\omega\left(\Phi_{u} X\right) \text { for all } X \in T M \text { and } u \in \eta
$$

Proof. Since $\xi$ and $\eta$ are flat, the same holds for $\gamma=\eta^{*} \otimes \xi$ by Lemma 3. The manifold $M^{n}$ being simply-connected, a one-form $\rho \in \Gamma\left(T^{*} M \otimes \gamma\right)$ is exact if and only if it is closed. 


\section{The Combescure transformation}

In this section we introduce a vectorial version of the Combescure transformation for submanifolds and derive a few properties of it that will be needed later.

Proposition 6. Let $f: M^{n} \rightarrow \mathbb{R}^{N}$ be an isometric immersion of a simply connected Riemannian manifold, let $V$ be a Euclidean vector space and let $\Phi \in$ $\Gamma\left(T^{*} M \otimes V^{*} \otimes T M\right)$. Then there exists $\mathcal{F} \in \Gamma\left(V^{*} \otimes f^{*} T \mathbb{R}^{N}\right)$ such that

$$
d \mathcal{F}(X)(v)=f_{*} \Phi_{v} X \text { for all } X \in T M \text { and } v \in V
$$

if and only if $\Phi$ is closed and satisfies

$$
\alpha\left(X, \Phi_{v} Y\right)=\alpha\left(Y, \Phi_{v} X\right) \text { for all } v \in \Gamma(V),
$$

where $\alpha: T M \times T M \rightarrow T^{\perp} M$ is the second fundamental form of $f$.

Proof. Applying (2) for $\omega=f_{*} \in \Gamma\left(T^{*} M \otimes f^{*} T \mathbb{R}^{N}\right)$ and $\Phi \in \Gamma\left(T^{*} M \otimes V^{*} \otimes T M\right)$ we obtain that the one-form $\rho=\rho\left(f_{*}, \Phi\right) \in \Gamma\left(T^{*} M \otimes V^{*} \otimes f^{*} T \mathbb{R}^{N}\right)$ satisfies

$$
\nabla \rho(X, Y) u=\alpha\left(X, \Phi_{u} Y\right)+f_{*}(\nabla \Phi(X, Y) u) .
$$

Therefore, $\Phi$ being closed and (4) are both necessary and sufficient conditions for $\rho$ to be closed. Since $V$ and $f^{*} T \mathbb{R}^{N}$ are flat, the result follows from Corollary 5 .

We call $\mathcal{F}$ a Combescure transform of $f$ determined by $\Phi$ if, in addition,

$$
\left\langle\Phi_{v} X, Y\right\rangle=\left\langle X, \Phi_{v} Y\right\rangle \text { for all } v \in \Gamma(V) .
$$

Observe that $\mathcal{F}$ is determined up to a parallel element in $\left.\Gamma\left(V^{*} \oplus f^{*} T \mathbb{R}^{N}\right)\right)$. Notice also that for each fixed vector $v \in V$, regarded as a parallel section of the trivial vector bundle $V$, we have that $\mathcal{F}(v) \in \Gamma\left(f^{*} T \mathbb{R}^{N}\right)$ satisfies $d \mathcal{F}(v)(X)=f_{*} \Phi_{v}(X)$, and hence $\mathcal{F}(v)$ is a Combescure transform of $f$ in the sense of [ 5 . determined by the Codazzi tensor $\Phi_{v}$.

Proposition 7. Let $f: M^{n} \rightarrow \mathbb{R}^{N}$ be an isometric immersion of a simply connected Riemannian manifold, let $V$ be a Euclidean vector space and let $\Phi \in$ $\Gamma\left(T^{*} M \otimes V^{*} \otimes T M\right)$ be closed and satisfy (4). For $\mathcal{F} \in \Gamma\left(V^{*} \otimes f^{*} T \mathbb{R}^{N}\right)$ satisfying (3) write

$$
\mathcal{F}=f_{*} \omega^{t}+\beta
$$

where $\omega \in \Gamma\left(T^{*} M \otimes V\right)$ and $\beta \in \Gamma\left(V^{*} \otimes T^{\perp} M\right)$. Then

$$
\alpha\left(X, \omega^{t}(v)\right)+\left(\nabla_{X}^{V^{*} \otimes T^{\perp} M} \beta\right) v=0 \text { for all } v \in \Gamma(V),
$$

and $\Phi$ is given by

$$
\Phi_{v} X=\left(\nabla_{X}^{V^{*} \otimes T M} \omega^{t}\right) v-A_{\beta(v)} X .
$$

Conversely, if $\omega \in \Gamma\left(T^{*} M \otimes V\right)$ and $\beta \in \Gamma\left(V^{*} \otimes T^{\perp} M\right)$ satisfy (17), then (3) holds for $\mathcal{F}=\mathcal{F}(\omega, \beta)$ and $\Phi=\Phi(\omega, \beta)$ given by (6) and (8), respectively. In particular, $\Phi$ is closed and (4) holds. Moreover, $\Phi$ satisfies (15) if and only if $\omega=d \varphi$ for some $\varphi \in \Gamma(V)$.

Proof. Denote by $\nabla^{*}$ the covariant derivative of $V^{*} \otimes f^{*} T \mathbb{R}^{N}$. Then,

$$
\begin{aligned}
d \mathcal{F}(X)(v) & =\left(\nabla_{X}^{*} f_{*} \omega^{t}\right) v+\left(\nabla_{X}^{*} \beta\right) v=\nabla_{X}^{f^{*} T \mathbb{R}^{N}} f_{*} \omega^{t}(v)-f_{*} \omega^{t}\left(\nabla_{X}^{V} v\right)+\left(\nabla_{X}^{*} \beta\right) v \\
& =f_{*} \nabla_{X} \omega^{t}(v)+\alpha\left(X, \omega^{t}(v)\right)-f_{*} \omega^{t}\left(\nabla_{X}^{V} v\right)+\left(\nabla_{X}^{V^{*} \otimes T^{\perp} M} \beta\right) v-f_{*} A_{\beta(v)} X .
\end{aligned}
$$

Since, on the other hand, $\mathcal{F}$ satisfies (3), then (7) and (8) follow. 
Conversely, if $\omega$ and $\beta$ satisfy (7), then the preceding computation yields (3) with $\Phi$ given by (8). Finally, taking the inner product of (8) with $Y \in \Gamma(T M)$ gives

$$
\left\langle\Phi_{v} X, Y\right\rangle=\langle v, \nabla \omega(X, Y)\rangle-\left\langle A_{\beta(v)} X, Y\right\rangle,
$$

thus the symmetry of $\nabla \omega$ is equivalent to (5).

Proposition 8. Let $f: M^{n} \rightarrow \mathbb{R}^{N}$ be an isometric immersion of a simply connected Riemannian manifold. Let $V_{i}, 1 \leq i \leq 2$, be Euclidean vector spaces, and assume that $\omega_{i} \in \Gamma\left(T^{*} M \otimes V_{i}\right)$ and $\beta_{i} \in \Gamma\left(V_{i}^{*} \otimes T^{\perp} M\right)$ satisfy

$$
\alpha\left(X, \omega_{i}^{t}\left(v_{i}\right)\right)+\left(\nabla_{X}^{V_{i}^{*} \otimes T^{\perp} M} \beta_{i}\right) v_{i}=0 \text { for all } v_{i} \in \Gamma\left(V_{i}\right) .
$$

Set $\mathcal{F}_{i}=f_{*} \omega_{i}^{t}+\beta_{i}$ and $\Phi_{v_{i}}^{i} X=\left(\nabla_{X}^{V_{i}^{*} \otimes T M} \omega_{i}^{t}\right) v_{i}-A_{\beta_{i}\left(v_{i}\right)} X$. Then,

$$
\nabla \omega_{i}\left(X, \Phi_{v_{j}}^{j} Y\right)=\nabla \omega_{i}\left(Y, \Phi_{v_{j}}^{j} X\right) \text { for all } v_{j} \in \Gamma\left(V_{j}\right)
$$

if and only if

$$
\left\langle\Phi_{v_{i}}^{i} X, \Phi_{v_{j}}^{j} Y\right\rangle=\left\langle\Phi_{v_{i}}^{i} Y, \Phi_{v_{j}}^{j} X\right\rangle \text { for all } v_{i} \in \Gamma\left(V_{i}\right) \text { and } v_{j} \in \Gamma\left(V_{j}\right) \text {. }
$$

When this is the case, there exists $\Omega_{i j}=\Omega\left(\omega_{i}, \Phi^{j}\right) \in \Gamma\left(V_{j}^{*} \otimes V_{i}\right)$ satisfying

$$
d \Omega_{i j}(X)\left(v_{j}\right)=\omega_{i}\left(\Phi_{v_{j}}^{j} X\right) \text { for all } v_{j} \in \Gamma\left(V_{j}\right) .
$$

In particular,

$$
d \Omega_{i j}=\mathcal{F}_{i}^{t} d \mathcal{F}_{j}
$$

and

$$
\Omega_{i j}+\Omega_{j i}^{t}=\mathcal{F}_{i}^{t} \mathcal{F}_{j}=\omega_{i} \omega_{j}^{t}+\beta_{i}^{t} \beta_{j}
$$

up to a parallel element in $\Gamma\left(V_{j}^{*} \otimes V_{i}\right)$.

Proof. Since $\omega_{j}$ and $\beta_{j}$ satisfy (9), we have $\alpha\left(X, \Phi_{v_{j}}^{j} Y\right)=\alpha\left(Y, \Phi_{v_{j}}^{j} X\right)$ by Proposition 7 Thus, it follows from

$$
\left\langle\Phi_{v_{i}}^{i} X, \Phi_{v_{j}}^{j} Y\right\rangle=\left\langle v_{i}, \nabla \omega_{i}\left(X, \Phi_{v_{j}}^{j} Y\right)\right\rangle-\left\langle\alpha\left(X, \Phi_{v_{j}}^{j} Y\right), \beta_{i}\left(v_{i}\right)\right\rangle
$$

that conditions (10) and (11) are equivalent. If (10) holds, then by Corollary 5 there exists $\Omega_{i j} \in \Gamma\left(V_{j}^{*} \otimes V_{i}\right)$ satisfying (12). On the other hand,

$$
\mathcal{F}_{i}^{t} d \mathcal{F}_{j}(X) v_{j}=\mathcal{F}_{i}^{t} f_{*} \Phi_{v_{j}}^{j} X=\omega_{i}\left(\Phi_{v_{j}}^{j} X\right) \text { for all } v_{j} \in \Gamma\left(V_{j}\right),
$$

and (13) follows. Finally, (13) implies that the exterior derivatives of both sides in the first equality of (14) coincide.

\section{The Vectorial Ribaucour transformation}

We now introduce the main concept of this paper.

Definition 9. Let $f: M^{n} \rightarrow \mathbb{R}^{N}$ be an isometric immersion of a simply connected Riemannian manifold, and let $V$ be a Euclidean vector space. Let $\varphi \in \Gamma(V)$ and $\beta \in \Gamma\left(V^{*} \otimes T^{\perp} M\right)$ satisfy (7) with $\omega=d \varphi$, and let $\Omega \in \Gamma(G l(V))$ be a solution of the completely integrable first order system

$$
d \Omega=\mathcal{F}^{t} d \mathcal{F}
$$

such that

$$
\Omega+\Omega^{t}=\mathcal{F}^{t} \mathcal{F}
$$


where $\mathcal{F}=f_{*} \omega^{t}+\beta$. If the map $\tilde{f}: M^{n} \rightarrow \mathbb{R}^{N}$ given by

$$
\tilde{f}=f-\mathcal{F} \Omega^{-1} \varphi
$$

is an immersion, then the isometric immersion $\tilde{f}: \tilde{M}^{n} \rightarrow \mathbb{R}^{N}$, where $\tilde{M}^{n}$ stands for $M^{n}$ with the metric induced by $\tilde{f}$, is called a vectorial Ribaucour transform of $f$ determined by $(\varphi, \beta, \Omega)$, and it is denoted by $\mathcal{R}_{\varphi, \beta, \Omega}(f)$.

Remark 10. If $\operatorname{dim} V=1$, after identifying $V^{*} \otimes T_{f}^{\perp} M$ with $T_{f}^{\perp} M$, then $\varphi$ and $\beta$ become elements of $C^{\infty}(M)$ and $\Gamma\left(T_{f}^{\perp} M\right)$, respectively, and (7) reduces to

$$
\alpha(X, \nabla \varphi)+\nabla_{X}^{\perp} \beta=0 .
$$

Moreover, $\Omega=(1 / 2)\langle\mathcal{F}, \mathcal{F}\rangle$ for $\mathcal{F}=f_{*} \nabla \varphi+\beta$, and (17) reduces to the parameterization of a scalar Ribaucour transform of $f$ obtained in Theorem 17 of [5]. In this case, since $\Omega$ is determined by $\varphi$ and $\beta$ we write $\tilde{f}=\mathcal{R}_{\varphi, \beta}(f)$ instead of $\tilde{f}=\mathcal{R}_{\varphi, \beta, \Omega}(f)$.

Next we derive several basic properties of the vectorial Ribaucour transformation.

Proposition 11. The bundle map $\mathcal{P} \in \Gamma\left(\left(f^{*} T \mathbb{R}^{N}\right)^{*} \otimes \tilde{f}^{*} T \mathbb{R}^{N}\right)$ given by

$$
\mathcal{P}=I-\mathcal{F} \Omega^{-1} \mathcal{F}^{t}
$$

is a vector bundle isometry and

$$
\tilde{f}_{*}=\mathcal{P} f_{*} D,
$$

where $D=I-\Phi_{\Omega^{-1} \varphi} \in \Gamma\left(T^{*} M \otimes T M\right)$. In particular, $\tilde{f}$ has the metric $\langle,\rangle^{\sim}=$ $D^{*}\langle$,$\rangle .$

Proof. We have

$$
\begin{aligned}
\mathcal{P}^{t} \mathcal{P} & =\left(I-\mathcal{F}\left(\Omega^{-1}\right)^{t} \mathcal{F}^{t}\right)\left(I-\mathcal{F} \Omega^{-1} \mathcal{F}^{t}\right) \\
& =I-\mathcal{F} \Omega^{-1} \mathcal{F}^{t}-\mathcal{F}\left(\Omega^{-1}\right)^{t} \mathcal{F}^{t}+\mathcal{F}\left(\Omega^{-1}\right)^{t} \mathcal{F}^{t} \mathcal{F} \Omega^{-1} \mathcal{F}^{t}
\end{aligned}
$$

Using (16) in the last term implies that the last three terms cancel out. Thus $\mathcal{P}$ is an isometry. Now, using (3) and (15) we obtain

$$
\begin{aligned}
\tilde{f}_{*} & =f_{*}-d \mathcal{F} \Omega^{-1} \varphi+\mathcal{F} \Omega^{-1} d \Omega \Omega^{-1} \varphi-\mathcal{F} \Omega^{-1} \omega \\
& =f_{*}-f_{*} \Phi_{\Omega^{-1} \varphi}+\mathcal{F} \Omega^{-1} \mathcal{F}^{t} d \mathcal{F} \Omega^{-1} \varphi-\mathcal{F} \Omega^{-1} \mathcal{F}^{t} f_{*} \\
& =f_{*}\left(I-\Phi_{\Omega^{-1} \varphi}\right)-\mathcal{F} \Omega^{-1} \mathcal{F}^{t} f_{*}\left(I-\Phi_{\Omega^{-1} \varphi}\right)=\mathcal{P} f_{*} D .
\end{aligned}
$$

Proposition 12. The normal connections and second fundamental forms of $f$ and $\tilde{f}$ are related by

$$
\tilde{\nabla}_{X}^{\perp} \xi=\mathcal{P} \nabla_{X}^{\perp} \xi
$$

and

$$
\tilde{A}_{\mathcal{P} \xi}=D^{-1}\left(A_{\xi}+\Phi_{\Omega^{-1} \beta^{t} \xi}\right)
$$

or equivalently,

$$
\tilde{\alpha}(X, Y)=\mathcal{P}\left(\alpha(X, D Y)+\beta\left(\Omega^{-1}\right)^{t} \Phi(X)^{t} D Y\right) .
$$


Proof. Let $\bar{\nabla}$ denote the connection of $\tilde{f}^{*} T \mathbb{R}^{N}$. Observing that $d \mathcal{F}^{t}(X)$ vanishes on $T^{\perp} M$, for $\left\langle d \mathcal{F}^{t}(X) \xi, v\right\rangle=\langle\xi, d \mathcal{F}(X) v\rangle=\left\langle\xi, f_{*} \Phi_{v}(X)\right\rangle=0$, and using (3), (15) and (19), we get

$$
\begin{aligned}
- & \tilde{f}_{*} \tilde{A}_{\mathcal{P} \xi} X+\tilde{\nabla}_{X}^{\perp} \xi=\bar{\nabla}_{X} \mathcal{P} \xi=\bar{\nabla}_{X}\left(\xi-\mathcal{F} \Omega^{-1} \mathcal{F}^{t} \xi\right) \\
= & -f_{*} A_{\xi} X+\nabla_{X}^{\perp} \xi-d \mathcal{F}(X) \Omega^{-1} \mathcal{F}^{t} \xi+\mathcal{F} \Omega^{-1} d \Omega(X) \Omega^{-1} \mathcal{F}^{t} \xi \\
& +\mathcal{F} \Omega^{-1} \mathcal{F}^{t}\left(f_{*} A_{\xi} X-\nabla_{X}^{\perp} \xi\right) \\
= & -\mathcal{P} f_{*} A_{\xi} X+\mathcal{P} \nabla_{X}^{\perp} \xi-\mathcal{P} f_{*} \Phi(X) \Omega^{-1} \mathcal{F}^{t} \xi,
\end{aligned}
$$

which gives (20) and (21).

Proposition 13. The triple $(\tilde{\varphi}, \tilde{\beta}, \tilde{\Omega})=\left(\Omega^{-1} \varphi, \mathcal{P} \beta\left(\Omega^{-1}\right)^{t}, \Omega^{-1}\right)$ satisfies the conditions of Definition 9 with respect to $\tilde{f}$, and $f=\mathcal{R}_{\tilde{\varphi}, \tilde{\beta}, \tilde{\Omega}}(\tilde{f})$. Moreover, $\tilde{\mathcal{F}}=$ $\tilde{f}_{*}(d \tilde{\varphi})^{t}+\tilde{\beta}$ and $\tilde{\Phi}=\Phi(d \tilde{\varphi}, \tilde{\beta})$ are given, respectively, by

$$
\tilde{\mathcal{F}}=-\mathcal{F} \Omega^{-1} \text { and } D \tilde{\Phi}_{v}=-\Phi_{\Omega^{-1} v} .
$$

Proof. Since $\tilde{\Omega}=d \tilde{\varphi}=-\Omega^{-1} \omega \Phi_{\Omega^{-1} \varphi}+\Omega^{-1} \varnothing=\Omega^{-1} \varnothing D$, we have

$$
\left\langle\tilde{\Omega}^{t}(v), X\right\rangle^{\sim}=\left\langle v, \Omega^{-1} \omega(D X)\right\rangle=\left\langle D \omega^{t}\left(\Omega^{-1}\right)^{t} v, X\right\rangle=\left\langle\omega^{t}\left(\Omega^{-1}\right)^{t} v, D^{-1} X\right\rangle^{\sim},
$$

thus

$$
D \tilde{\Omega}^{t}=\omega^{t}\left(\Omega^{-1}\right)^{t},
$$

where we have used that $D^{-1}$ is symmetric with respect to $\langle,\rangle \sim$. We now prove that

$$
\tilde{\alpha}\left(X, \tilde{\Omega}^{t}(v)\right)+\left(\nabla_{X}^{V^{*} \otimes T_{\tilde{f}}^{\perp} \tilde{M}} \tilde{\beta}\right) v=0 \text { for all } v \in \Gamma(V) .
$$

Equations (22) and (24) yield

$$
\tilde{\alpha}\left(X, \tilde{\Omega}^{t}(v)\right)=\mathcal{P}\left(\alpha\left(X, \omega^{t}\left(\Omega^{-1}\right)^{t} v\right)+\beta\left(\Omega^{-1}\right)^{t} \Phi(X)^{t} \omega^{t}\left(\Omega^{-1}\right)^{t} v\right),
$$

whereas (20) gives

$$
\begin{aligned}
\left(\nabla_{X}^{V^{*} \otimes T_{\tilde{f}}^{\perp} \tilde{M}} \tilde{\beta}\right) v & =\tilde{\nabla} \frac{\perp}{X} \tilde{\beta}(v)-\tilde{\beta}\left(\nabla_{X}^{V} v\right)=\mathcal{P}\left(\nabla_{X}^{\perp} \beta\left(\Omega^{-1}\right)^{t} v-\beta\left(\Omega^{-1}\right)^{t} \nabla_{X}^{V} v\right) \\
& =\mathcal{P}\left(\left(\nabla_{X}^{V^{*} \otimes T^{\perp} M} \beta\right)\left(\Omega^{-1}\right)^{t} v-\beta\left(\Omega^{-1}\right)^{t} \Phi(X)^{t} \omega^{t}\left(\Omega^{-1}\right)^{t} v\right) .
\end{aligned}
$$

It follows from (7), (26) and (27) that (25) holds.

We now compute $\tilde{\mathcal{F}}=\tilde{f}_{*} \tilde{\Omega}^{t}+\tilde{\beta}$. Using (19) in the first equality below, (24) in the second and (16) in the last one, we obtain (28)

$\tilde{\mathcal{F}}=\mathcal{P} f_{*} D \tilde{\Omega}^{t}+\tilde{\beta}=\mathcal{P}\left(f_{*} \omega^{t}\left(\Omega^{-1}\right)^{t}+\beta\left(\Omega^{-1}\right)^{t}\right)=\left(I-\mathcal{F} \Omega^{-1} \mathcal{F}^{t}\right) \mathcal{F}\left(\Omega^{-1}\right)^{t}=-\mathcal{F} \Omega^{-1}$.

Then, it follows from (15), (16) and (28) that

$$
\tilde{\mathcal{F}}^{t} d \tilde{\mathcal{F}}=\left(\Omega^{-1}\right)^{t} \mathcal{F}^{t} d \mathcal{F} \Omega^{-1}-\left(\Omega^{-1}\right)^{t} \mathcal{F}^{t} \mathcal{F} \Omega^{-1} d \Omega \Omega^{-1}=d \tilde{\Omega},
$$

and

$$
\tilde{\mathcal{F}}^{t} \tilde{\mathcal{F}}=\left(\Omega^{-1}\right)^{t} \mathcal{F}^{t} \mathcal{F} \Omega^{-1}=\tilde{\Omega}+\tilde{\Omega}^{t} .
$$

Therefore,

$$
\mathcal{R}_{\tilde{\varphi}, \tilde{\beta}, \tilde{\Omega}}(\tilde{f})=\tilde{f}-\tilde{\mathcal{F}} \tilde{\Omega}^{-1} \tilde{\varphi}=f-\mathcal{F} \Omega^{-1} \varphi-\left(-\mathcal{F} \Omega^{-1}\right) \Omega \Omega^{-1} \varphi=f .
$$


Finally, the second formula in (23) follows from

$$
\begin{aligned}
\tilde{f}_{*} \tilde{\Phi}_{v}(X) & =d \tilde{\mathcal{F}}(X) v=-d \mathcal{F}(X) \Omega^{-1} v+\mathcal{F} \Omega^{-1} d \Omega(X) \Omega^{-1} v \\
& =-f_{*} \Phi_{\Omega^{-1} v}(X)+\mathcal{F} \Omega^{-1} \omega \Phi_{\Omega^{-1}}(X)=-\mathcal{P} f_{*} \Phi_{\Omega^{-1} v}(X) \\
& =-\tilde{f}_{*} D^{-1} \Phi_{\Omega^{-1} v}(X) .
\end{aligned}
$$

\section{The DECOMPOSITION THEOREM}

A fundamental feature of the vectorial Ribaucour transformation for submanifolds is the following decomposition property, first proved in [13] in the context of orthogonal systems.

Theorem 14. Let $\mathcal{R}_{\varphi, \beta, \Omega}(f): \tilde{M}^{n} \rightarrow \mathbb{R}^{N}$ be a vectorial Ribaucour transform of an isometric immersion $f: M^{n} \rightarrow \mathbb{R}^{N}$. For an orthogonal decomposition $V=V_{1} \oplus V_{2}$ define

(29) $\varphi_{j}=\pi_{V_{j}} \circ \varphi, \quad \beta_{j}=\left.\beta\right|_{V_{j}} \quad$ and $\quad \Omega_{i j}=\left.\pi_{V_{i}} \circ \Omega\right|_{V_{j}} \in \Gamma\left(V_{j}^{*} \otimes V_{i}\right), \quad 1 \leq i, j \leq 2$.

Assume that $\Omega_{j j}$ is invertible and, for $i \neq j$, define $\mathcal{R}_{\varphi, \beta, \Omega}\left(\varphi_{i}, \beta_{i}, \Omega_{i i}\right)=\left(\bar{\varphi}_{i}, \bar{\beta}_{i}, \bar{\Omega}_{i i}\right)$ by

$$
\bar{\varphi}_{i}=\varphi_{i}-\Omega_{i j} \Omega_{j j}^{-1} \varphi_{j}, \quad \bar{\beta}_{i}=\mathcal{P}_{j}\left(\beta_{i}-\beta_{j}\left(\Omega_{j j}^{-1}\right)^{t} \Omega_{i j}^{t}\right) \quad \text { and } \quad \bar{\Omega}_{i i}=\Omega_{i i}-\Omega_{i j} \Omega_{j j}^{-1} \Omega_{j i},
$$

where $\mathcal{P}_{j}=I-\mathcal{F}_{j} \Omega_{j j}^{-1} \mathcal{F}_{j}^{t}$. Then the triples $\left(\varphi_{j}, \beta_{j}, \Omega_{j j}\right)$ and $\left(\bar{\varphi}_{i}, \bar{\beta}_{i}, \bar{\Omega}_{i i}\right)$ satisfy the conditions of Definition 9 with respect to $f$ and $f_{j}$, respectively, and we have

$$
\mathcal{R}_{\varphi, \beta, \Omega}(f)=\mathcal{R}_{\bar{\varphi}_{i}, \bar{\beta}_{i}, \bar{\Omega}_{i i}}\left(\mathcal{R}_{\varphi_{j}, \beta_{j}, \Omega_{j j}}(f)\right) .
$$

Proof. That $\left(\varphi_{j}, \beta_{j}, \Omega_{j j}\right), 1 \leq j \leq 2$, satisfies the conditions of Definition 9 with respect to $f$ is clear. In order to prove that $\left(\bar{\varphi}_{i}, \bar{\beta}_{i}, \bar{\Omega}_{i i}\right)$ satisfies the conditions of Definition 9 with respect to $f_{j}$ for $i \neq j$ we first compute $\bar{\omega}_{i}=d \bar{\varphi}_{i}$. We have

$$
\begin{aligned}
\bar{\omega}_{i}(X) & =\omega_{i}(X)+-d \Omega_{i j}(X) \Omega_{j j}^{-1} \varphi_{j}+\Omega_{i j} \Omega_{j j}^{-1} d \Omega_{j j}(X) \Omega_{j j}^{-1} \varphi_{j}-\Omega_{i j} \Omega_{j j}^{-1} \omega_{j}(X) \\
& =\omega_{i}(X)-\omega_{i}\left(\Phi^{j}(X) \Omega_{j j}^{-1} \varphi_{j}\right)+\Omega_{i j} \Omega_{j j}^{-1} \omega_{j}\left(\Phi^{j}(X) \Omega_{j j}^{-1} \varphi_{j}\right)-\Omega_{i j} \Omega_{j j}^{-1} \omega_{j}(X) \\
& =\omega_{i}\left(D_{j} X\right)-\Omega_{i j} \Omega_{j j}^{-1} \omega_{j}\left(D_{j} X\right),
\end{aligned}
$$

where $D_{j}=I-\Phi_{\Omega_{j j}^{-1} \varphi_{j}}^{j}$. Then

$$
\begin{aligned}
\left\langle\bar{\omega}_{i}^{t}\left(v_{i}\right), X\right\rangle_{j} & =\left\langle v_{i}, \omega_{i}\left(D_{j} X\right)-\Omega_{i j} \Omega_{j j}^{-1} \omega_{j}\left(D_{j} X\right)\right\rangle \\
& =\left\langle D_{j} \omega_{i}^{t}\left(v_{i}\right)-D_{j} \omega_{j}^{t}\left(\Omega_{j j}^{-1}\right)^{t} \Omega_{i j}^{t}\left(v_{i}\right), X\right\rangle \\
& =\left\langle\omega_{i}^{t}\left(v_{i}\right)-\omega_{j}^{t}\left(\Omega_{j j}^{-1}\right)^{t} \Omega_{i j}^{t}\left(v_{i}\right), D_{j}^{-1} X\right\rangle_{j},
\end{aligned}
$$

where $\langle,\rangle_{j}$ denotes the metric induced by $f_{j}$. Using that $D_{j}^{-1}$ is symmetric with respect to $\langle,\rangle_{j}$, we obtain that

$$
D_{j} \bar{\omega}_{i}^{t}=\omega_{i}^{t}-\omega_{j}^{t}\left(\Omega_{j j}^{-1}\right)^{t} \Omega_{i j}^{t} .
$$

It follows from (22) that

$$
\begin{aligned}
\alpha_{j}\left(X, \bar{\omega}_{i}^{t}\left(v_{i}\right)\right) & =\mathcal{P}_{j}\left(\alpha\left(X, \omega_{i}^{t}\left(v_{i}\right)\right)-\alpha\left(X, \omega_{j}^{t}\left(\Omega_{j j}^{-1}\right)^{t} \Omega_{i j}^{t}\left(v_{i}\right)\right)+\beta_{j}\left(\Omega_{j j}^{-1}\right)^{t} \Phi^{j}(X)^{t} \omega_{i}^{t}\left(v_{i}\right)\right. \\
& \left.+\beta_{j}\left(\Omega_{j j}^{-1}\right)^{t} \Phi^{j}(X)^{t} \omega_{j}^{t}\left(\Omega_{j j}^{-1}\right)^{t} \Omega_{i j}^{t}\left(v_{i}\right)\right)
\end{aligned}
$$


where $\alpha_{j}$ is the second fundamental form of $f_{j}$. On the other hand, we obtain from

$$
\begin{aligned}
& \left(\nabla_{X} \bar{\beta}_{i}\right)\left(v_{i}\right)=\nabla_{X}^{\perp} \bar{\beta}_{i}\left(v_{i}\right)-\bar{\beta}_{i}\left(\nabla_{X}^{V} v_{i}\right) \\
& \quad=\mathcal{P}_{j}\left(\nabla_{X}^{\perp} \beta_{i}\left(v_{i}\right)-\nabla_{X}^{\perp} \beta_{j}\left(\Omega_{j j}^{-1}\right)^{t} \Omega_{i j}^{t}\left(v_{i}\right)-\beta_{i}\left(\nabla_{X}^{V} v_{i}\right)+\beta_{j}\left(\Omega_{j j}^{-1}\right)^{t} \Omega_{i j}^{t}\left(\nabla_{X}^{V} v_{i}\right)\right)
\end{aligned}
$$

and

$$
\begin{aligned}
& -\nabla_{X}^{\frac{1}{X}} \beta_{j}\left(\Omega_{j j}^{-1}\right)^{t} \Omega_{i j}^{t}\left(v_{i}\right)+\beta_{j}\left(\Omega_{j j}^{-1}\right)^{t} \Omega_{i j}^{t}\left(\nabla_{X} v_{i}\right) \\
& \quad=-\left(\nabla_{X} \beta_{j}\right)\left(\left(\Omega_{j j}^{-1}\right)^{t} \Omega_{i j}^{t}\left(v_{i}\right)\right)-\beta_{j}\left(d\left(\Omega_{j j}^{-1}\right)^{t}(X) \Omega_{i j}^{t}\left(v_{i}\right)\right)-\beta_{j}\left(\Omega_{j j}^{-1}\right)^{t} d \Omega_{i j}^{t}(X)\left(v_{i}\right)
\end{aligned}
$$

that

$$
\begin{aligned}
\left(\nabla_{X} \bar{\beta}_{i}\right)\left(v_{i}\right)= & \mathcal{P}_{j}\left(\left(\nabla_{X} \beta_{i}\right)\left(v_{i}\right)-\left(\nabla_{X} \beta_{j}\right)\left(\left(\Omega_{j j}^{-1}\right)^{t} \Omega_{i j}^{t}\left(v_{i}\right)\right)-\beta_{j}\left(\Omega_{j j}^{-1}\right)^{t} \Phi^{j}(X)^{t} \omega_{i}^{t}\left(v_{i}\right)\right. \\
& \left.+\beta_{j}\left(\Omega_{j j}^{-1}\right)^{t} \Phi^{j}(X)^{t} \omega_{j}^{t}\left(\Omega_{j j}^{-1}\right)^{t} \Omega_{i j}^{t}\left(v_{i}\right)\right),
\end{aligned}
$$

where we used $d \Omega_{i j}^{t}(X)=\Phi^{j}(X)^{t} \omega_{i}^{t}$. It follows from (30) and (31) that

$$
\alpha_{j}\left(X, \bar{\omega}_{i}^{t}\left(v_{i}\right)\right)+\left(\nabla_{X} \bar{\beta}_{i}\right)\left(v_{i}\right)=0 .
$$

Now we have

$$
\begin{aligned}
\overline{\mathcal{F}}_{i} & =f_{j_{*}} \bar{\omega}_{i}^{t}+\bar{\beta}_{i}=\mathcal{P}_{j} f_{*} D_{j} \bar{\omega}_{i}^{t}+\mathcal{P}_{j}\left(\beta_{i}-\beta_{j}\left(\Omega_{j j}^{-1}\right)^{t} \Omega_{i j}^{t}\right) \\
& =\mathcal{P}_{j}\left(f_{*} \omega_{i}^{t}+\beta_{i}-f_{*} \omega_{j}^{t}\left(\Omega_{j j}^{-1}\right)^{t} \Omega_{i j}^{t}-\beta_{j}\left(\Omega_{j j}^{-1}\right)^{t} \Omega_{i j}^{t}=\mathcal{P}_{j}\left(\mathcal{F}_{i}-\mathcal{F}_{j}\left(\Omega_{j j}^{-1}\right)^{t} \Omega_{i j}^{t}\right)\right. \\
& =\mathcal{F}_{i}-\mathcal{F}_{j}\left(\Omega_{j j}^{-1}\right)^{t} \Omega_{i j}^{t}-\mathcal{F}_{j} \Omega_{j j}^{-1} \mathcal{F}_{j}^{t} \mathcal{F}_{i}+\mathcal{F}_{j} \Omega_{j j}^{-1} \mathcal{F}_{j}^{t} \mathcal{F}_{j}\left(\Omega_{j j}^{-1}\right)^{t} \Omega_{i j}^{t} \\
& =\mathcal{F}_{i}-\mathcal{F}_{j} \Omega_{j j}^{-1} \Omega_{j i},
\end{aligned}
$$

where we used that $\mathcal{F}_{j}^{t} \mathcal{F}_{i}=\Omega_{j i}+\Omega_{i j}^{t}$. Then,

$$
\begin{aligned}
\overline{\mathcal{F}}_{i}^{t} d \overline{\mathcal{F}}_{i}=\overline{\mathcal{F}}_{i}^{t}\left(d \mathcal{F}_{i}-d \mathcal{F}_{j} \Omega_{j j}^{-1} \Omega_{j i}+\mathcal{F}_{j} \Omega_{j j}^{-1} d \Omega_{j j} \Omega_{j j}^{-1} \Omega_{j i}-\mathcal{F}_{j} \Omega_{j j}^{-1} d \Omega_{j i}\right) \\
=\mathcal{F}_{i}^{t} d \mathcal{F}_{i}-\mathcal{F}_{i}^{t} d \mathcal{F}_{j} \Omega_{j j}^{-1} \Omega_{j i}+\mathcal{F}_{i}^{t} \mathcal{F}_{j} \Omega_{j j}^{-1} d \Omega_{j j} \Omega_{j j}^{-1} \Omega_{j i}-\mathcal{F}_{i}^{t} \mathcal{F}_{j} \Omega_{j j}^{-1} d \Omega_{j i} \\
\quad-\Omega_{j i}^{t}\left(\Omega_{j j}^{-1}\right)^{t} \mathcal{F}_{j}^{t} d \mathcal{F}_{i}+\Omega_{j i}^{t}\left(\Omega_{j j}^{-1}\right)^{t} \mathcal{F}_{j}^{t} d \mathcal{F}_{j} \Omega_{j j}^{-1} \Omega_{j i} \\
-\Omega_{j i}^{t}\left(\Omega_{j j}^{-1}\right)^{t} \mathcal{F}_{j}^{t} \mathcal{F}_{j} \Omega_{j j}^{-1} d \Omega_{j j} \Omega_{j j}^{-1} \Omega_{j i}+\Omega_{j i}^{t}\left(\Omega_{j j}^{-1}\right)^{t} \mathcal{F}_{j}^{t} \mathcal{F}_{j} \Omega_{j j}^{-1} d \Omega_{j i} .
\end{aligned}
$$

Using that $d \Omega_{j i}=\mathcal{F}_{j}^{t} d \mathcal{F}_{i}$ and $d \Omega_{j j}=\mathcal{F}_{j}^{t} d \mathcal{F}_{j}$, we obtain

$$
\overline{\mathcal{F}}_{i}^{t} d \overline{\mathcal{F}}_{i}=d \Omega_{i i}-d \Omega_{i j} \Omega_{j j}^{-1} \Omega_{j i}+\Omega_{i j} \Omega_{j j}^{-1} d \Omega_{j j} \Omega_{j j}^{-1} \Omega_{j i}-\Omega_{i j} \Omega_{j j}^{-1} d \Omega_{j i}=d \bar{\Omega}_{i i} .
$$

Moreover,

$$
\begin{aligned}
\overline{\mathcal{F}}_{i}^{t} \overline{\mathcal{F}}_{i} & =\left(\mathcal{F}_{i}^{t}-\Omega_{j i}^{t}\left(\Omega_{j j}^{-1}\right)^{t} \mathcal{F}_{j}^{t}\right)\left(\mathcal{F}_{i}-\mathcal{F}_{j} \Omega_{j j}^{-1} \Omega_{j i}\right) \\
& =\mathcal{F}_{i}^{t} \mathcal{F}_{i}-\mathcal{F}_{i}^{t} \mathcal{F}_{j} \Omega_{j j}^{-1} \Omega_{j i}-\Omega_{j i}^{t}\left(\Omega_{j j}^{-1}\right)^{t} \mathcal{F}_{j}^{t} \mathcal{F}_{i}+\Omega_{j i}^{t}\left(\Omega_{j j}^{-1}\right)^{t} \mathcal{F}_{j}^{t} \mathcal{F}_{j} \Omega_{j j}^{-1} \Omega_{j i} \\
& =\Omega_{i i}-\Omega_{i j} \Omega_{j j}^{-1} \Omega_{j i}+\Omega_{i i}^{t}-\Omega_{j i}^{t}\left(\Omega_{j j}^{-1}\right)^{t} \Omega_{i j}^{t}=\bar{\Omega}_{i i}+\bar{\Omega}_{i i}^{t},
\end{aligned}
$$

which completes the proof that $\left(\bar{\varphi}_{i}, \bar{\beta}_{i}, \bar{\Omega}_{i i}\right)$ satisfies the required conditions.

Now write $\Omega$ in matrix notation as

$$
\Omega=\left(\begin{array}{ll}
\Omega_{11} & \Omega_{12} \\
\Omega_{21} & \Omega_{22}
\end{array}\right) .
$$

Since $\Omega$ and $\Omega_{i i}$ are invertible, then $\bar{\Omega}_{i i}$ is invertible for $1 \leq i \leq 2$ and

$$
\Omega^{-1}=\left(\begin{array}{cc}
\bar{\Omega}_{11}^{-1} & -\bar{\Omega}_{11}^{-1} \Omega_{12} \Omega_{22}^{-1} \\
-\bar{\Omega}_{22}^{-1} \Omega_{21} \Omega_{11}^{-1} & \bar{\Omega}_{22}^{-1}
\end{array}\right) .
$$


In particular,

$$
\bar{\Omega}_{i i}^{-1}=\Omega_{i i}^{-1}+\Omega_{i i}^{-1} \Omega_{i j} \bar{\Omega}_{j j}^{-1} \Omega_{j i} \Omega_{i i}^{-1} \quad \text { and } \quad \bar{\Omega}_{i i}^{-1} \Omega_{i j} \Omega_{j j}^{-1}=\Omega_{i i}^{-1} \Omega_{i j} \bar{\Omega}_{j j}^{-1}
$$

for $1 \leq i \neq j \leq 2$. Then,

$$
\begin{aligned}
\mathcal{R}_{\varphi, \beta, \Omega}(f) & =f-\mathcal{F} \Omega^{-1} \varphi \\
& =f-\mathcal{F}\left(\bar{\Omega}_{11}^{-1}\left(\varphi_{1}-\Omega_{12} \Omega_{22}^{-1} \varphi_{2}\right)+\bar{\Omega}_{22}^{-1}\left(\varphi_{2}-\Omega_{21}\left(\Omega_{11}\right)^{-1} \varphi_{1}\right)\right) \\
& =f-\mathcal{F}_{1} \bar{\Omega}_{11}^{-1}\left(\varphi_{1}-\Omega_{12} \Omega_{22}^{-1} \varphi_{2}\right)-\mathcal{F}_{2} \bar{\Omega}_{22}^{-1}\left(\varphi_{2}-\Omega_{21} \Omega_{11}^{-1} \varphi_{1}\right) .
\end{aligned}
$$

On the other hand, by (32) and (32) we have

$$
\begin{aligned}
\mathcal{R}_{\bar{\varphi}_{i}, \bar{\beta}_{i}, \bar{\Omega}_{i i}}\left(f_{j}\right) & =f_{j}-\overline{\mathcal{F}}_{i} \bar{\Omega}_{i i} \bar{\varphi}_{i} \\
& =f-\mathcal{F}_{j} \Omega_{j j}^{-1} \varphi_{j}-\left(\mathcal{F}_{i}-\mathcal{F}_{j} \Omega_{j j}^{-1} \Omega_{j i}\right) \bar{\Omega}_{i i}^{-1}\left(\varphi_{i}-\Omega_{i j} \Omega_{j j}^{-1} \varphi_{j}\right) \\
& =f-\left(\mathcal{F}_{j} \bar{\Omega}_{j j}^{-1}-\mathcal{F}_{i} \bar{\Omega}_{i i}^{-1} \Omega_{i j} \Omega_{j j}^{-1}\right) \varphi_{j}-\left(\mathcal{F}_{i} \bar{\Omega}_{i i}^{-1}-\mathcal{F}_{j} \bar{\Omega}_{j j}^{-1} \Omega_{j i} \Omega_{i i}^{-1}\right) \varphi_{i} .
\end{aligned}
$$

We conclude that $\mathcal{R}_{\varphi, \beta, \Omega}(f)=\mathcal{R}_{\bar{\varphi}_{i}, \bar{\beta}_{i}, \bar{\Omega}_{i i}}\left(f_{j}\right)$ for $1 \leq i \neq j \leq 2$.

Remark 15. It follows from Theorem 14 that a vectorial Ribaucour transformation whose associated data $(\varphi, \beta, \Omega)$ are defined on a vector space $V$ can be regarded as the iteration of $k=\operatorname{dim} V$ scalar Ribaucour transformations.

In applying Theorem 14 it is often more convenient to use one of its two following consequences.

Corollary 16. Let $f_{i}=\mathcal{R}_{\varphi_{i}, \beta_{i}, \Omega_{i i}}(f): M_{i}^{n} \rightarrow \mathbb{R}^{N}, 1 \leq i \leq 2$, be two vectorial Ribaucour transforms of $f: M^{n} \rightarrow \mathbb{R}^{N}$. Assume that the tensors $\Phi^{i}=\Phi\left(d \varphi_{i}, \beta_{i}\right)$ satisfy

$$
\left[\Phi_{v_{i}}^{i}, \Phi_{v_{j}}^{j}\right]=0 \text { for all } v_{i} \in V_{i} \text { and } v_{j} \in V_{j}, \quad 1 \leq i \neq j \leq 2 .
$$

Set $\mathcal{F}_{i}=f_{*}\left(d \varphi_{i}\right)^{t}+\beta_{i}$. Then there exists $\Omega_{i j} \in \Gamma\left(V_{j}^{*} \otimes V_{i}\right)$, such that

$$
d \Omega_{i j}=\mathcal{F}_{i}^{t} d \mathcal{F}_{j} \quad \text { and } \quad \mathcal{F}_{i}^{t} \mathcal{F}_{j}=\Omega_{i j}+\Omega_{j i}^{t},
$$

and such that $\varphi \in \Gamma(V), \beta \in \Gamma\left(V^{*} \otimes T^{\perp} M\right)$ and $\Omega \in \Gamma\left(V^{*} \otimes V\right)$ defined by (29) for $V=V_{1} \oplus V_{2}$ satisfy the conditions of definition 9 (and therefore the remaining conclusions of Theorem 14 hold).

Proof. The first assertion is a consequence of Proposition 8 . It is now easily seen that $\varphi \in \Gamma(V), \beta \in \Gamma\left(V^{*} \otimes T^{\perp} M\right), \Omega \in \Gamma\left(V^{*} \otimes V\right)$ defined by (29) for $V=V_{1} \oplus V_{2}$ satisfy the conditions of Definition 9 with respect to $f$ if and only if the same holds for $\left(\varphi_{i}, \beta_{i}, \Omega_{i i}\right)$ and, in addition, (33) holds.

Corollary 17. Let $f_{1}=\mathcal{R}_{\varphi_{1}, \beta_{1}, \Omega_{11}}(f): \tilde{M}^{n} \rightarrow \mathbb{R}^{N}$ be a vectorial Ribaucour transform of $f: M^{n} \rightarrow \mathbb{R}^{N}$. Let $\left(\bar{\varphi}_{2}, \bar{\beta}_{2}, \bar{\Omega}_{22}\right)$ satisfy the conditions of Definition 9 with respect to $f_{1}$. Assume further that $\bar{\Phi}^{2}=\Phi\left(d \bar{\varphi}_{2}, \bar{\beta}_{2}\right)$ satisfies

$$
\left[\bar{\Phi}_{v_{2}}^{2}, \bar{\Phi}_{v_{1}}^{1}\right]=0, \text { for all } v_{1} \in V_{1}, v_{2} \in V_{2},
$$

where $D_{1} \bar{\Phi}_{v_{1}}^{1}=-\Phi_{\Omega_{11}^{-1} v_{1}}^{1}$ for $\Phi^{1}=\Phi\left(d \varphi_{1}, \beta_{1}\right)$. Then there exist $\bar{\Omega}_{i j} \in \Gamma\left(V_{j}^{*} \otimes V_{i}\right)$, $i \neq j$, such that

$$
d \bar{\Omega}_{i j}=\overline{\mathcal{F}}_{i}^{t} d \overline{\mathcal{F}}_{j} \quad \text { and } \quad \overline{\mathcal{F}}_{i}^{t} \overline{\mathcal{F}}_{j}=\bar{\Omega}_{i j}+\bar{\Omega}_{j i}^{t},
$$


where $\overline{\mathcal{F}}_{1}=-\mathcal{F}_{1} \Omega_{11}^{-1}$ and $\overline{\mathcal{F}}_{2}=\left(f_{1}\right)_{*}\left(d \bar{\varphi}_{2}\right)^{t}+\bar{\beta}_{2}$. Now define

$$
\begin{gathered}
\left(\varphi_{2}, \beta_{2}, \Omega_{22}\right)=\left(\bar{\varphi}_{2}-\bar{\Omega}_{21} \varphi_{1}, \mathcal{P}_{1}^{-1} \bar{\beta}_{2}-\beta_{1} \bar{\Omega}_{21}^{t}, \bar{\Omega}_{22}-\bar{\Omega}_{21} \Omega_{11} \bar{\Omega}_{12}\right), \\
\Omega_{12}=\Omega_{11} \bar{\Omega}_{12} \text { and } \Omega_{21}=-\bar{\Omega}_{21} \Omega_{11} .
\end{gathered}
$$

Then $\varphi \in \Gamma(V), \beta \in \Gamma\left(V^{*} \otimes T^{\perp} M\right)$ and $\Omega \in \Gamma\left(V^{*} \otimes V\right)$ defined by (29) for $V=V_{1} \oplus$ $V_{2}$ satisfy the conditions of Definition 9 and $\mathcal{R}_{\varphi, \beta, \Omega}(f)=\mathcal{R}_{\bar{\varphi}_{2}, \bar{\beta}_{2}, \bar{\Omega}_{22}}\left(\mathcal{R}_{\varphi_{1}, \beta_{1}, \Omega_{11}}(f)\right)$.

Proof. By Proposition 13 , we have $f=\mathcal{R}_{\bar{\varphi}_{1}, \bar{\beta}_{1}, \bar{\Omega}_{11}}\left(f_{1}\right)$ where

$$
\left(\bar{\varphi}_{1}, \bar{\beta}_{1}, \bar{\Omega}_{11}\right)=\left(\Omega_{11}^{-1} \varphi_{1}, \mathcal{P}_{1} \beta_{1}\left(\Omega_{11}^{-1}\right)^{t}, \Omega_{11}^{-1}\right) .
$$

Moreover, $\overline{\mathcal{F}}_{1}=\left(f_{1}\right)_{*}\left(d \bar{\varphi}_{1}\right)^{t}+\bar{\beta}_{1}$ and $\bar{\Phi}_{1}=\Phi\left(d \bar{\varphi}_{1}, \bar{\beta}_{1}\right)$ are given by

$$
\overline{\mathcal{F}}_{1}=-\mathcal{F}_{1} \Omega_{11}^{-1} \text { and } D_{1} \bar{\Phi}_{v_{1}}^{1}=-\Phi_{\Omega_{11}^{-1} v_{1}}^{1} .
$$

Thus, the existence of $\bar{\Omega}_{i j} \in \Gamma\left(V_{j}^{*} \otimes V_{i}\right), i \neq j$, satisfying condition (34) follows from Proposition 8 applied to $f_{1}$ and the triples $\left(\bar{\varphi}_{i}, \bar{\beta}_{i}, \bar{\Omega}_{i i}\right), 1 \leq i \leq 2$. Now observe that $\left(\varphi_{2}, \beta_{2}, \Omega_{22}\right)=\mathcal{R}_{\bar{\varphi}, \bar{\beta}, \bar{\Omega}}\left(\bar{\varphi}_{2}, \bar{\beta}_{2}, \bar{\Omega}_{2}\right)$, and hence $\left(\varphi_{2}, \beta_{2}, \Omega_{22}\right)$ satisfies (7) with respect to $f_{1}$ and $d \Omega_{22}=\mathcal{F}_{2}^{t} d \mathcal{F}_{2}$ by Theorem 14 .

It remains to check that $d \Omega_{i j}=\mathcal{F}_{i}^{t} d \mathcal{F}_{j}$ and $\Omega_{i j}+\Omega_{j i}^{t}=\mathcal{F}_{i}^{t} \mathcal{F}_{j}, \leq i \neq j$. From the proof of Theorem 14(see (32)) we have $\mathcal{F}_{2}=\overline{\mathcal{F}}_{2}-\overline{\mathcal{F}}_{1} \bar{\Omega}_{11}^{-1} \bar{\Omega}_{12}=\overline{\mathcal{F}}_{2}-\overline{\mathcal{F}}_{1} \Omega_{11} \bar{\Omega}_{12}$. Then,

$$
\begin{aligned}
\mathcal{F}_{2}^{t} d \mathcal{F}_{1} & =-\left(\overline{\mathcal{F}}_{2}^{t}-\bar{\Omega}_{12}^{t} \Omega_{11}^{t} \overline{\mathcal{F}}_{1}^{t} d\left(\overline{\mathcal{F}}_{1} \Omega_{11}\right)\right)=-\overline{\mathcal{F}}_{2}^{t} d \overline{\mathcal{F}}_{1} \Omega_{11}-\overline{\mathcal{F}}_{2}^{t} \overline{\mathcal{F}}_{1} d \Omega_{11}+\bar{\Omega}_{12}^{t} \mathcal{F}_{1}^{t} d \mathcal{F}_{1} \\
& =-d \bar{\Omega}_{21} \Omega_{11}-\left(\bar{\Omega}_{21}+\bar{\Omega}_{12}^{t}\right) d \Omega_{11}+\bar{\Omega}_{12}^{t} d \Omega_{11}-d\left(\bar{\Omega}_{21} \Omega_{11}\right) \\
& =d \Omega_{21} .
\end{aligned}
$$

A similar computation shows that $\mathcal{F}_{1}^{t} d \mathcal{F}_{2}=\Omega_{12}$.

Finally, we have

$$
\begin{aligned}
\mathcal{F}_{1}^{t} \mathcal{F}_{2} & =-\Omega_{11}^{t} \overline{\mathcal{F}}_{1}^{t}\left(\overline{\mathcal{F}}_{2}-\overline{\mathcal{F}}_{1} \bar{\Omega}_{11}^{-1} \bar{\Omega}_{12}\right)=-\Omega_{11}^{t}\left(\bar{\Omega}_{12}+\bar{\Omega}_{21}^{t}-\left(\bar{\Omega}_{11}+\bar{\Omega}_{11}^{t}\right) \bar{\Omega}_{11}^{-1} \bar{\Omega}_{12}\right) \\
& =-\Omega_{11}^{t}\left(\bar{\Omega}_{21}^{t}-\bar{\Omega}_{11}^{t} \Omega_{11} \bar{\Omega}_{12}\right)=-\Omega_{11}^{t} \bar{\Omega}_{21}^{t}+\Omega_{11} \bar{\Omega}_{12}=\Omega_{11} \bar{\Omega}_{12}-\left(\bar{\Omega}_{21} \Omega_{11}\right)^{t} \\
& =\Omega_{12}+\Omega_{21}^{t}
\end{aligned}
$$

and similarly one checks that $\mathcal{F}_{2}^{t} \mathcal{F}_{1}=\Omega_{21}+\Omega_{12}^{t}$.

Given four submanifolds $f_{i}: M_{i}^{n} \rightarrow \mathbb{R}^{N}, 1 \leq i \leq 4$, we say that they form a Bianchi quadrilateral if for each of them both the preceding and subsequent ones (thought of as points on an oriented circle) are Ribaucour transforms of it, and the Codazzi tensors associated to the transformations commute.

Proof of Theorem 2, We first prove existence. Write $f_{i}=\mathcal{R}_{\varphi_{i}, \beta_{i}}(f), 1 \leq i \leq k$. For each pair $\{i, j\} \subset\{1, \ldots, k\}$ with $i<j$ define $\varphi^{i j} \in \Gamma\left(\mathbb{R}^{2}\right)$ and

$$
\beta^{i j} \in \Gamma\left(\left(\mathbb{R}^{2}\right)^{*} \otimes T^{\perp} M\right)
$$

by

$$
\varphi^{i j}=\left(\varphi_{i}, \varphi_{j}\right) \text { and } \beta^{i j}=d x_{1} \otimes \beta_{i}+d x_{2} \otimes \beta_{j} .
$$

By the assumption that $\left\{f_{i j}, f_{i}, f_{j}, f\right\}$ is a Bianchi quadrilateral, then $\Omega^{i j} \in G l\left(\mathbb{R}^{2}\right)$ with

$$
\Omega^{i j}\left(e_{1}\right)=\Omega_{i i}=(1 / 2)\left\langle\mathcal{F}_{i}, \mathcal{F}_{i}\right\rangle \quad \text { and } \quad \Omega^{i j}\left(e_{2}\right)=\Omega_{j j}=(1 / 2)\left\langle\mathcal{F}_{j}, \mathcal{F}_{j}\right\rangle
$$


where $\mathcal{F}_{r}=f_{*} \nabla \varphi_{r}+\beta_{r}, r \in\{i, j\}$, such that $\left(\varphi^{i j}, \beta^{i j}, \Omega^{i j}\right)$ satisfies the conditions of Definition 9 with respect to $f$ and such that $f_{i j}=\mathcal{R}_{\varphi^{i j}, \beta^{i j}, \Omega^{i j}}(f)$. Define $\varphi \in \Gamma\left(\mathbb{R}^{k}\right)$, $\beta \in \Gamma\left(\left(\mathbb{R}^{k}\right)^{*} \otimes T^{\perp} M\right)$ and $\Omega \in \Gamma\left(\left(\mathbb{R}^{k}\right)^{*} \otimes \mathbb{R}^{k}\right)$ by

$$
\varphi=\left(\varphi_{1}, \ldots, \varphi_{k}\right), \quad \beta=\sum_{i=1}^{k} d x_{i} \otimes \beta_{i}
$$

and

$$
\Omega=\sum_{i=1}^{k} \Omega_{i i} d x_{i} \otimes e_{i}+\sum_{i<j}\left(\left\langle\Omega^{i j}\left(e_{1}\right), e_{2}\right\rangle d x_{i} \otimes e_{j}+\left\langle\Omega^{i j}\left(e_{2}\right), e_{1}\right\rangle d x_{j} \otimes e_{i}\right) .
$$

It is easy to check that $(\varphi, \beta, \Omega)$ satisfies the conditions of Definition 9 with respect to $f$.

We now make precise the "generic" assumption on the statement of Theorem 2 , Namely, we require that no principal minor of $\Omega$ vanishes, where $\Omega$ is regarded as a square $(k \times k)$-matrix. That is, for any multi-index $\alpha=\left\{i_{1}<\ldots<i_{r}\right\} \subset\{1, \ldots, k\}$, the sub-matrix $\Omega_{\alpha}$ of $\Omega$, formed by those elements of $\Omega$ that belong to the rows and columns with indexes in $\alpha$, has nonzero determinant. Now, for any such $\alpha$ set

$$
\varphi^{\alpha}=\left(\varphi_{i_{1}}, \ldots, \varphi_{i_{r}}\right), \quad \beta^{\alpha}=\sum_{j=1}^{r} d x_{i_{j}} \otimes \beta_{i_{j}} \quad \text { and } \quad \Omega^{\alpha}=\Omega_{\alpha} .
$$

We define $\mathcal{C}_{r}$ as the family of $\left(\begin{array}{l}k \\ r\end{array}\right)$ elements formed by the vectorial Ribaucour transforms $\mathcal{R}_{\varphi^{\alpha}, \beta^{\alpha}, \Omega^{\alpha}}(f)$, where $\alpha$ ranges on the set of multi-indexes $\alpha=\left\{i_{1}<\right.$ $\left.\ldots<i_{r}\right\} \subset\{1, \ldots, k\}$ with $r$ elements. Given

$\hat{f}=\mathcal{R}_{\varphi^{\alpha}, \beta^{\alpha}, \Omega^{\alpha}}(f) \in \mathcal{C}_{s+1}, \quad 1 \leq s \leq k-1$ and $\alpha=\left\{i_{1}<\ldots<i_{s+1}\right\} \subset\{1, \ldots, k\}$,

let $\alpha_{1}, \ldots, \alpha_{s+1}$ be the $(s+1)$ multi-indexes with $s$ elements that are contained in $\alpha$. For each $j=1, \ldots, s+1$ write $\alpha=\alpha_{j} \cup\left\{i_{j}\right\}$. Then,

$$
\hat{f}_{j}:=\mathcal{R}_{\varphi^{\alpha_{j}}, \beta^{\alpha_{j}}, \Omega^{\alpha_{j}}}(f) \in \mathcal{C}_{s} \text { and } \hat{f}=\mathcal{R}_{\bar{\varphi}_{i_{j}}, \bar{\beta}_{i_{j}}}\left(\hat{f}_{j}\right)
$$

by Theorem 14. Therefore $\hat{f}$ is a Ribaucour transform of $\hat{f}_{1}, \ldots, \hat{f}_{s+1}$. Moreover,

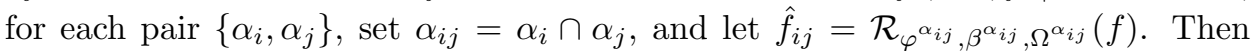
$\hat{f}_{i j} \in \mathcal{C}_{s-1}$ and $\left\{\hat{f}_{i j}, \hat{f}_{i}, \hat{f}_{j}, \hat{f}\right\}$ is a Bianchi quadrilateral

Next we argue for the uniqueness. We first make precise the meaning of $f_{1}, \ldots, f_{k}$ being independent Ribaucour transforms of $f$. Namely, if $f_{i}$ is determined by the pair $\left(\varphi_{i}, \beta_{i}\right)$ with $\varphi_{i} \in C^{\infty}(M)$ and $\beta_{i} \in \Gamma\left(T_{f}^{\perp} M\right), 1 \leq i \leq k$, we require that the image of the map $\varphi=\left(\varphi_{1}, \ldots, \varphi_{k}\right): M \rightarrow \mathbb{R}^{k}$ spans $\mathbb{R}^{k}$ and, in addition, that the linear map $\mathcal{F}: \mathbb{R}^{k} \rightarrow f^{*} T \mathbb{R}^{N}$ given by $\mathcal{F}=\sum_{i=1}^{k} d x_{i} \otimes \mathcal{F}_{i}$, with $\mathcal{F}_{i}=f_{*} \nabla \varphi_{i}+\beta_{i}$, is injective.

It is easily seen that all uniqueness assertions follow from the uniqueness for $k=3$. For this case, the independence assumption is equivalent to the condition that none of $f_{1}, f_{2}$ or $f_{3}$ belong to the associated family determined by the other two. Then, uniqueness was proved in [11] by using a nice elementary argument relying on the version of Miquel's Theorem for four circumferences. 


\section{Submanifolds CARRYing A PARALlEL FLAT NORMAL SUBBUNDLE}

In this section we give an explicit local construction of all submanifolds of Euclidean space that carry a parallel flat normal subbundle, from which Theorem 1 in the introduction follows as a special case.

Theorem 18. Let $f: M^{n} \rightarrow \mathbb{R}^{n+p}$ be an isometric immersion of a simply connected Riemannian manifold and let $\varphi_{i} \in C^{\infty}(M)$ and $\beta_{i} \in \Gamma\left(T^{\perp} M\right), 1 \leq i \leq m$, satisfy

$$
\alpha\left(X, \nabla \varphi_{i}\right)+\nabla_{X}^{\perp} \beta_{i}=0
$$

and

$$
\left[\Phi_{i}, \Phi_{j}\right]=0, \quad 1 \leq i, j \leq m,
$$

where $\Phi_{i}=\operatorname{Hess} \varphi_{i}-A_{\beta_{i}}$. Define $\mathcal{G}: M^{n} \rightarrow M_{(n+p) \times m}(\mathbb{R})$ by

$$
\mathcal{G}=\left(f_{*} \nabla \varphi_{1}+\beta_{1}, \ldots, f_{*} \nabla \varphi_{m}+\beta_{m}\right) .
$$

Then there exists a smooth map $\Omega: U \rightarrow G L\left(\mathbb{R}^{m}\right)$ on an open subset $U \subset M^{n}$ such that

$$
d \Omega=\mathcal{G}^{t} d \mathcal{G} \quad \text { and } \quad \Omega+\Omega^{t}=\mathcal{G}^{t} \mathcal{G}+I .
$$

Moreover, the map $\tilde{f}: \tilde{M}^{n} \rightarrow \mathbb{R}^{n+p+m}$ given by

$$
\tilde{f}(p)=\left(\begin{array}{c}
f(p)^{t}-\mathcal{G} \Omega^{-1} \varphi(p) \\
-\Omega^{-1} \varphi(p)
\end{array}\right)
$$

where $\varphi(p)=\left(\varphi_{1}(p), \ldots, \varphi_{m}(p)\right)^{t}$, defines, on an open subset $\tilde{M}^{n} \subset U$ of regular points, an immersion carrying a parallel flat normal subbundle of rank $\mathrm{m}$.

Conversely, any isometric immersion carrying a parallel flat normal subbundle of rank $m$ can be locally constructed in this way.

Proof. Set $V=\mathbb{R}^{m}$ and define $\beta \in \Gamma\left(V^{*} \oplus T^{\perp} M\right)$ by $\beta\left(e_{i}\right)=\beta_{i}+e_{i}$, where $\left\{e_{i}\right\}_{1 \leq i \leq m}$ is the canonical basis of $\mathbb{R}^{m}$ regarded as the orthogonal complement of $\mathbb{R}^{n+p}$ in $\mathbb{R}^{n+p+m}$. Then $\omega=d \varphi$ and $\beta$ satisfy (17) in view of (35). Moreover, $\mathcal{F} \in \Gamma\left(V^{*} \oplus f^{*} T \mathbb{R}^{n+m+p}\right)$ given by $\mathcal{F}(v)=f_{*} \omega^{t}(v)+\beta(v)$ satisfies

$$
\mathcal{F}=\left(\begin{array}{c}
\mathcal{G} \\
I_{m}
\end{array}\right)
$$

where $I_{m}$ denotes the $m \times m$ identity matrix. Thus

$$
\mathcal{F}^{t} d \mathcal{F}=\mathcal{G}^{t} d \mathcal{G} \text { and } \mathcal{F}^{t} \mathcal{F}=\mathcal{G}^{t} \mathcal{G}+I_{m},
$$

and the existence of $\Omega$ satisfying (37) follows from Proposition 8 by using (36). Moreover, comparing (17) and (38) we have that $\tilde{f}=\mathcal{R}_{\varphi, \beta, \Omega}(f)$. Since $\mathbb{R}^{m}$ is a parallel flat normal vector subbundle of $T^{\perp} M$ (where $f$ is regarded as an immersion into $\left.\mathbb{R}^{n+p+m}\right)$ and $\mathcal{P} \in \Gamma\left(\left(f^{*} T \mathbb{R}^{N}\right)^{*} \otimes \tilde{f}^{*} T \mathbb{R}^{N}\right)$ given by (18) is a parallel vector bundle isometry by virtue of (20), it follows that $\mathcal{P}\left(\mathbb{R}^{m}\right)$ is a parallel flat normal vector subbundle of $T_{\tilde{f}} M$ of rank $m$.

In order to prove the converse, it suffices to show that, given an isometric immersion $f: M^{n} \rightarrow \mathbb{R}^{n+p+m}$ carrying a parallel flat normal subbundle $E$ of rank $m$, there exist locally an immersion $\tilde{f}: M^{n} \rightarrow \mathbb{R}^{n+p} \subset \mathbb{R}^{n+p+m}$ and $\tilde{\varphi} \in \Gamma\left(V:=\mathbb{R}^{m}\right)$, $\tilde{\beta} \in \Gamma\left(V^{*} \oplus T_{\tilde{f}}^{\perp} M\right)$ and $\tilde{\Omega} \in \Gamma(G l(V))$ satisfying the conditions of Definition 9 such that $\left(\tilde{\beta}\left(e_{i}\right)\right)_{\mathbb{R}^{m}}=e_{i}, 1 \leq i \leq m$, and that $f=\mathcal{R}_{\tilde{\varphi}, \tilde{\beta}, \tilde{\Omega}}(\tilde{f})$. 
Let $\xi_{1}, \ldots, \xi_{m}$ be an orthonormal parallel frame of $E$. Let $V=\mathbb{R}^{m}$ be identified with a subspace of $\mathbb{R}^{N}$ and let $e_{1}, \ldots, e_{m}$ be the canonical basis of $\mathbb{R}^{m}$. Define $\varphi \in \Gamma(V)$ and $\beta \in \Gamma\left(V^{*} \otimes T^{\perp} M\right)$ by

$$
\varphi=-\sum_{i=1}^{m}\left\langle f, e_{i}\right\rangle e_{i} \text { and } \beta(v)=\sum_{i=1}^{m} x_{i}\left(\xi_{i}-e_{i}^{\perp}\right),
$$

for $v=\left(x_{1}, \ldots, x_{m}\right)$, where $e_{i}^{\perp}$ denotes the normal vector field obtained by orthogonally projecting $e_{i}$ pointwise onto $T^{\perp} M$. Then

$$
\omega^{t}(v)=-\sum_{i=1}^{m} x_{i} f_{*}^{t} e_{i} \quad \text { and } \quad \mathcal{F}(v)=\sum_{i=1}^{m} x_{i}\left(\xi_{i}-e_{i}\right) .
$$

Therefore,

$$
\begin{aligned}
\alpha\left(X, \omega^{t}(v)\right)+\left(\nabla_{X}^{V^{*} \otimes T^{\perp} M} \beta\right) v & =-\sum_{i=1}^{m} x_{i} \alpha\left(X, f_{*}^{t} e_{i}\right)+\sum_{i=1}^{m} x_{i} \nabla_{X}^{\perp}\left(\xi_{i}-e_{i}^{\perp}\right) \\
& =-\sum_{i=1}^{m} x_{i}\left(\tilde{\nabla}_{X}\left(f_{*}^{t} e_{i}+e_{i}^{\perp}\right)\right)^{\perp}=\sum_{i=1}^{m} x_{i}\left(\tilde{\nabla}_{X} e_{i}\right)^{\perp}=0
\end{aligned}
$$

where $\tilde{\nabla}$ denotes the Euclidean connection, and hence (7) is satisfied.

It also follows from (39) that

$$
\left\langle\mathcal{F}^{t} \xi_{j}, v\right\rangle=\left\langle\xi_{j}, \mathcal{F}(v)\right\rangle=x_{j}-\sum_{i=1}^{m} x_{i}\left\langle\xi_{j}, e_{i}\right\rangle
$$

thus $\mathcal{F}^{t} \xi_{j}=e_{j}-\sum_{i=1}^{m}\left\langle\xi_{j}, e_{i}\right\rangle e_{i}$. Similarly, $\mathcal{F}^{t} e_{j}=-e_{j}+\sum_{i=1}^{m}\left\langle\xi_{i}, e_{j}\right\rangle e_{i}$. We obtain

$$
\mathcal{F}^{t} \mathcal{F}(v)=\sum_{j=1}^{m} x_{j} \mathcal{F}^{t}\left(\xi_{j}-e_{j}\right)=\sum_{j=1}^{m} 2 x_{j} e_{j}-\sum_{i, j=1}^{m} x_{j}\left(\left\langle\xi_{j}, e_{i}\right\rangle+\left\langle\xi_{i}, e_{j}\right\rangle\right) e_{i}
$$

In matrix notation, this reads as

$$
\mathcal{F}^{t} \mathcal{F}=2 I-\left(\left\langle\xi_{j}, e_{i}\right\rangle\right)-\left(\left\langle\xi_{i}, e_{j}\right\rangle\right) .
$$

Therefore $\Omega=I-\left(\left\langle\xi_{i}, e_{j}\right\rangle\right)$ satisfies (15) and (16). Moreover, since

$$
\Omega e_{j}=e_{j}-\sum_{i=1}^{m}\left\langle\xi_{j}, e_{i}\right\rangle e_{i}=\mathcal{F}^{t} \xi_{j}
$$

we have

$$
\mathcal{P} \xi_{j}=\xi_{j}-\mathcal{F} \Omega^{-1} \mathcal{F}^{t} \xi_{j}=\xi_{j}-\mathcal{F} e_{j}=\xi_{j}-\left(\xi_{j}-e_{j}\right)=e_{j} .
$$

Therefore $\tilde{f}=\mathcal{R}_{\varphi, \beta, \Omega}(f)$ is such that $\tilde{f}\left(M^{n}\right)$ is contained in an affine subspace orthogonal to $\mathbb{R}^{m}$. Since $f=\mathcal{R}_{\tilde{\varphi}, \tilde{\beta}, \tilde{\Omega}}(\tilde{f})$ with the triple $(\tilde{\varphi}, \tilde{\beta}, \tilde{\Omega})$ given by Proposition [13, in order to complete the proof of the theorem it remains to show that $\left(\tilde{\beta}\left(e_{i}\right)\right)_{\mathbb{R}^{m}}=e_{i}$. But this follows from

$\left\langle\tilde{\beta} e_{i}, e_{j}\right\rangle=\left\langle\mathcal{P} \beta\left(\Omega^{-1}\right)^{t} e_{i}, e_{j}\right\rangle=\left\langle e_{i}, \Omega^{-1} \mathcal{F}^{t} \mathcal{P}^{t} e_{j}\right\rangle=\left\langle e_{i}, \Omega^{-1} \mathcal{F}^{t} \xi_{j}\right\rangle=\left\langle e_{i}, e_{j}\right\rangle$.

The case of submanifolds with a flat normal bundle of the sphere now follows easily from Theorem 1 
Corollary 19. Let $U \subset \mathbb{R}^{n},\left\{\varphi_{i}\right\}_{1 \leq i \leq m}, \mathcal{G}: U \rightarrow M_{n \times m}(\mathbb{R})$, and $\Omega: V \subset U \rightarrow$ $G l\left(\mathbb{R}^{m}\right)$ be as in Theorem 1 . Then the $M_{(n+m) \times m}(\mathbb{R})$-valued map

$$
W=\left(\begin{array}{c}
\mathcal{G} \Omega^{-1} \\
I-\Omega^{-1}
\end{array}\right)
$$

satisfies $W^{t} W=I$ and any of its columns defines, at regular points, the position vector of an immersion with flat normal bundle into $\mathbb{S}^{n+m-1}$.

Conversely, any isometric immersion with flat normal bundle $f: M^{n} \rightarrow \mathbb{S}^{n+m-1}$ can be locally constructed in this way.

Proof. Set $V=\mathbb{R}^{m}$ and define $\beta \in \Gamma\left(V^{*} \oplus T^{\perp} M\right)$ by $\beta\left(e_{i}\right)=e_{i}$, where $\left\{e_{i}\right\}_{1 \leq i \leq m}$ is the canonical basis of $\mathbb{R}^{m}$ regarded as the orthogonal complement of $\mathbb{R}^{n+p}$ in $\mathbb{R}^{n+p+m}$. Then $\omega=d \varphi$ and $\beta$ trivially satisfy (7). Moreover, if

$$
\mathcal{F} \in \Gamma\left(V^{*} \oplus f^{*} T \mathbb{R}^{n+m+p}\right)
$$

is given by $\mathcal{F}(v)=f_{*} \omega^{t}(v)+\beta(v)$, then

$$
\mathcal{F}=\left(\begin{array}{c}
\mathcal{G} \\
I_{m}
\end{array}\right)
$$

where $I_{m}$ denotes the $m \times m$ identity matrix. Let $\tilde{f}=\mathcal{R}_{\varphi, \beta, \Omega}(i d)$, where $i d$ is the inclusion of $U$ into $\mathbb{R}^{n}$. Then the isometry $\mathcal{P}$ as in (18) is given by

$$
\left(\begin{array}{cc}
I-\mathcal{G} \Omega^{-1} \mathcal{G}^{t} & \mathcal{G} \Omega^{-1} \\
\Omega^{-1} \mathcal{G}^{t} & I-\Omega^{-1}
\end{array}\right) .
$$

Therefore $W^{t} W=I$ and the $(n+p+j)^{t h}$-column of $W$ is $\mathcal{P} e_{j}, 1 \leq j \leq m$. Therefore it is a unit parallel normal vector field to $f$, and hence defines, at regular points, the position vector of a submanifold with flat normal bundle of $\mathbb{S}^{n+m-1}$.

The converse follows from the converse in Theorem 1 and the fact that any isometric immersion $f: M^{n} \rightarrow \mathbb{S}^{n+m-1}$ arises as a parallel unit normal vector field of an isometric immersion $F: M^{n} \rightarrow \mathbb{R}^{n+m}$, for instance, $F=i \circ f$, where $i$ is the canonical inclusion of $\mathbb{S}^{n+m-1}$ into $\mathbb{R}^{n+m}$.

We now give a precise statement of Ferapontov's theorem referred to in the introduction for the case of holonomic submanifolds of the sphere, and show how it can be derived from Corollary 19 .

Theorem 20. On an open simply connected subset $U \subset \mathbb{R}^{n}$ let $\left\{\beta_{i j}\right\}_{1 \leq i \neq j \leq n}$ be smooth real functions satisfying the completely integrable system of PDE's

$$
\left\{\begin{array}{l}
\frac{\partial \beta_{i j}}{\partial u_{k}}=\beta_{i k} \beta_{k j}, \quad 1 \leq i \neq j \neq k \neq i \leq n, \\
\frac{\partial \beta_{i j}}{\partial u_{i}}+\frac{\partial \beta_{j i}}{\partial u_{j}}+\sum_{k} \beta_{k i} \beta_{k j}=0, \quad i \neq j,
\end{array}\right.
$$

let $H^{\alpha}=\left(H_{1}^{\alpha}, \ldots, H_{n}^{\alpha}\right), 1 \leq \alpha \leq m$, be arbitrary solutions of the linear system of $P D E$ 's

$$
\frac{\partial H_{j}}{\partial u_{i}}=\beta_{i j} H_{i}, \quad 1 \leq i \neq j \leq n,
$$


and let $X_{i}: U \rightarrow \mathbb{R}^{n}, 1 \leq i \leq n$, satisfy

$$
\frac{\partial X_{i}}{\partial u_{j}}=\beta_{i j} X_{j}, \quad i \neq j, \quad \frac{\partial X_{i}}{\partial u_{i}}=-\sum_{k \neq i} \beta_{k i} X_{k}
$$

and $X^{t} X=I$ at some point of $U$, where $X=\left(X_{1}, \ldots, X_{n}\right)$, the integrability conditions of (41) and (42) being satisfied by virtue of (40). Then there exist vector functions $s^{\alpha}=\left(s_{1}^{\alpha}, \ldots, s_{n}^{\alpha}\right): U \rightarrow \mathbb{R}^{n}, 1 \leq \alpha \leq m$, such that $d s_{i}^{\alpha}=\sum_{k=1}^{n} X_{i k} H_{k}^{\alpha} d u^{k}$, and a map $\Omega: U \rightarrow M_{n \times m}(\mathbb{R})$ such that

$$
d \Omega=\mathcal{G}^{t} d \mathcal{G} \text { and } \Omega+\Omega^{t}=\mathcal{G}^{t} \mathcal{G}+I_{m},
$$

where $\mathcal{G}=\left(s^{1}, \ldots, s^{m}\right)$. Moreover, the $(m+n) \times m$-matrix

$$
W=\left(\begin{array}{c}
\mathcal{G} \Omega^{-1} \\
\cdots \\
I_{m}-\Omega^{-1}
\end{array}\right)
$$

satisfies $W^{t} W=I_{m}$ and any of its columns defines, at regular points, the position vector of an $n$-dimensional submanifold $M^{n} \subset \mathbb{S}^{n+m-1} \subset \mathbb{R}^{n+m}$ with flat normal bundle such that $u_{1}, \ldots, u_{n}$ are principal coordinates of $M^{n}$.

Conversely, any $n$-dimensional submanifold with flat normal bundle of $\mathbb{S}^{n+m-1}$ carrying a holonomic net of curvature lines can be locally constructed in this way.

Proof. It is easily checked using (42), and the fact that $X^{t} X=I$ at some point of $U$, that $X^{t} X=I$ everywhere on $U$, whence $X_{1}, \ldots, X_{n}$ determine an orthonormal frame on $U$. Define $\Phi^{\alpha} \in \Gamma\left(T^{*} U \otimes T U\right)$ by

$$
\Phi^{\alpha} X_{i}=H_{i}^{\alpha} X_{i}, \quad 1 \leq i \leq n, 1 \leq \alpha \leq m .
$$

Then $\Phi^{\alpha}$ is a symmetric tensor and, by (41) and (42),

$$
\frac{\partial}{\partial u_{j}}\left(\Phi^{\alpha} X_{i}\right)=\frac{\partial H_{i}^{\alpha}}{\partial u_{j}} X_{i}+H_{i}^{\alpha} \frac{\partial X_{i}}{\partial u_{j}}=\beta_{j i} H_{j}^{\alpha} X_{i}+H_{i}^{\alpha} \beta_{i j} X_{j}=\frac{\partial}{\partial u_{i}}\left(\Phi^{\alpha} X_{j}\right),
$$

hence $\Phi^{\alpha}$ is a Codazzi tensor on $U$. Thus $\Phi^{\alpha}$ is closed as a one-form in $U$ with values in $T U$. Since $U$ is flat, there exists $Z^{\alpha} \in \Gamma(T U)$ such that $\Phi^{\alpha}=d Z^{\alpha}$. Moreover, the symmetry of $\Phi^{\alpha}$ implies that $Z^{\alpha}=\operatorname{grad} \varphi^{\alpha}$ for some $\varphi^{\alpha} \in C^{\infty}(U)$, and hence $\Phi^{\alpha}=$ Hess $\varphi^{\alpha}$ (cf. [7]). Since $\left\{X_{i}\right\}_{1 \leq i \leq n}$ is a common diagonalizing basis of Hess $\varphi^{\alpha}, 1 \leq \alpha \leq m$, it follows that [Hess $\varphi_{\alpha}$, Hess $\left.\varphi_{\beta}\right]=0,1 \leq \alpha, \beta \leq m$. Setting $s^{\alpha}=\operatorname{grad} \varphi^{\alpha}$, the remainder of the proof follows from Corollary 19 .

Remark 21. Equations (40) (Lamé equations) and (41) are well-known in the theory of $n$-orthogonal systems (cf. [3]) where the functions $H_{i}$ and $\beta_{i j}$ are usually called the Lamé and rotation coefficients, respectively.

\section{Submanifolds CARRYing a DUPIN PRINCIPAL NORMAL}

A smooth normal vector field $\eta$ of an isometric immersion $f: M^{n} \rightarrow \mathbb{R}^{N}$ is called a principal normal with multiplicity $m \geq 1$ if the tangent subspaces

$$
\mathcal{E}_{\eta}=\operatorname{ker}(\alpha-\langle,\rangle \eta)
$$

have constant dimension $m \geq 1$. If $\eta$ is parallel in the normal connection along the nullity distribution $\mathcal{E}_{\eta}$, then $\eta$ is said to be a Dupin principal normal. This 
condition is automatic if $m \geq 2$. If $\eta$ is nowhere vanishing, it is well-known that $\mathcal{E}_{\eta}$ is an involutive distribution whose leaves are round $m$-dimensional spheres in $\mathbb{R}^{N}$. When $\eta$ vanishes identically, the distribution $\mathcal{E}_{\eta}=\mathcal{E}_{0}$ is known as the relative nullity distribution, in which case the leaves are open subsets of affine subspaces of $\mathbb{R}^{N}$.

Let $h: L^{n-m} \rightarrow \mathbb{R}^{N}$ be an isometric immersion carrying a parallel flat normal subbundle $\mathcal{N}$ of rank $m$, and let $\varphi \in C^{\infty}\left(L^{n-m}\right)$ and $\beta \in \Gamma\left(T_{h}^{\perp} L\right)$ satisfy

$$
\alpha(X, \nabla \varphi)+\nabla_{X}^{\perp} \beta=0 .
$$

Assume further that the tangent subspaces

$$
E(x)=\left\{Z \in T_{x} L:(\alpha(Z, X))_{\mathcal{N}^{\perp}}=\varphi^{-1} \beta_{\mathcal{N}^{\perp}}\langle Z, X\rangle \text { for all } X \in T_{x} L\right\}
$$

are everywhere trivial. Define $\mathcal{R}_{\varphi, \beta}^{\mathcal{N}}(h): \mathcal{N} \rightarrow \mathbb{R}^{N}$ by

$$
\mathcal{R}_{\varphi, \beta}^{\mathcal{N}}(h)(t)=\mathcal{R}_{\varphi, \beta+t^{\prime}}(h)(x),
$$

where $x=\pi(t)$ and $t^{\prime}$ is the parallel section in $\mathcal{N}$ such that $t^{\prime}(x)=t$. It was shown in [6] that $\mathcal{R}_{\varphi, \beta}^{\mathcal{N}}(h)$ defines, at regular points, an immersion carrying a Dupin principal normal with integrable conullity distribution $\mathcal{E}_{\eta}^{\perp}$ and that, conversely, any such immersion can be locally constructed in this way.

Using the results of the previous sections we now give an explicit description of all isometric immersions carrying a Dupin principal normal of multiplicity $m$ and integrable conullity in terms of the vectorial Ribaucour transformation, starting with an isometric immersion $g: L^{n-m} \rightarrow \mathbb{R}^{N}$ such that $g\left(L^{n-m}\right)$ lies in an $(N-m)$ dimensional subspace $\mathbb{R}^{N-m} \subset \mathbb{R}^{N}$.

Namely, let $\mathcal{R}_{\varphi, \beta, \Omega}(g)$ be a vectorial Ribaucour transform of an isometric immersion $g: L^{n-m} \rightarrow \mathbb{R}^{N-m} \subset \mathbb{R}^{N}$ determined by $(\varphi, \beta, \Omega)$ as in Definition 9 . For an orthogonal decomposition $\mathbb{R}^{m+1}=\mathbb{R} \oplus \mathbb{R}^{m}$, with $\mathbb{R}=\operatorname{span}\left\{e_{0}\right\}$, set

$$
\left(\varphi_{0}, \beta_{0}\right)=\left(\left\langle\varphi, e_{0}\right\rangle, \beta\left(e_{0}\right)\right), \quad\left(\varphi_{1}, \beta_{1}\right)=\left(\pi_{\mathbb{R}^{m}} \circ \varphi,\left.\beta\right|_{\mathbb{R}^{m}}\right),
$$

and

$$
\left(\bar{\varphi}_{0}, \hat{\beta}_{0}\right)=\left(\varphi_{0}-\Omega_{01} \Omega_{11}^{-1} \varphi_{1}, \beta_{0}-\beta_{1}\left(\Omega_{11}^{-1}\right)^{t} \Omega_{01}^{t} e_{0}\right)
$$

where

$$
\Omega_{11}=\left.\pi_{\mathbb{R}^{m}} \circ \Omega\right|_{\mathbb{R}^{m}} \text { and } \quad \Omega_{01}=\left.\pi_{\mathbb{R}} \circ \Omega\right|_{\mathbb{R}^{m}} .
$$

Assume that the bilinear maps $\gamma: T_{x} L \times T_{x} L \rightarrow T_{x} L^{\perp}$ given by

$$
\gamma(Z, X)=\left(\alpha_{g}\left(Z, D_{1} X\right)+\beta_{1}\left(\Omega_{11}^{-1}\right)^{t} \Phi_{1}(Z) D_{1}(X)-\bar{\varphi}_{0}\langle Z, X\rangle \hat{\beta}_{0}\right)_{\mathbb{R}^{N-m}}
$$

have an everywhere trivial kernel. Let the subspace $\mathbb{R}^{m}=e_{0}^{\perp}$ be identified with the orthogonal complement of $\mathbb{R}^{N-m}$ in $\mathbb{R}^{N}$ and choose an orthonormal basis $\left\{e_{0}, \ldots, e_{m}\right\}$ of $V:=\mathbb{R}^{m+1}$. Finally, for $t=\sum_{i=1}^{m} t_{i} e_{i} \in \mathbb{R}^{m}$ define

$$
\beta_{t}=e_{0}^{*} \otimes\left(\beta_{0}+t\right)+\sum_{i=1}^{m} e_{i}^{*} \otimes \beta\left(e_{i}\right) \quad \text { and } \quad \Omega_{t}=\Omega+\left(\left\langle\beta_{0}, t\right\rangle+(1 / 2)|t|^{2}\right) e_{0}^{*} \otimes e_{0} .
$$

Theorem 22. The triple $\left(\varphi, \beta_{t}, \Omega_{t}\right)$ satisfies the conditions of Definition 9 with respect to $g$ for each $t \in \mathbb{R}^{m}$ and the map $G: L^{n-m} \times \mathbb{R}^{m} \rightarrow \mathbb{R}^{N}$ given by

$$
G(x, t)=\mathcal{R}_{\varphi, \beta_{t}, \Omega_{t}}(g)(x)
$$


parameterizes, at regular points, an n-dimensional submanifold carrying a Dupin principal normal of multiplicity $m$ with integrable conullity.

Conversely, any isometric immersion carrying a Dupin principal normal of multiplicity $m$ with integrable conullity can be locally constructed in this way.

Proof. The first assertion is easily checked. By Theorem 14 we have

$$
\mathcal{R}_{\varphi, \beta_{t}, \Omega_{t}}(g)=\mathcal{R}_{\bar{\varphi}_{0}, \bar{\beta}_{t}^{0}}\left(\mathcal{R}_{\varphi_{1}, \beta_{1}, \Omega_{11}}(g)\right)
$$

where

$$
\bar{\beta}_{t}^{0}=\mathcal{P}_{1}\left(\beta_{0}+t-\beta_{1}\left(\Omega_{11}^{-1}\right)^{t} \Omega_{01}^{t} e_{0}\right)=\bar{\beta}^{0}+\sum_{i=1}^{m} t_{i} \eta_{i},
$$

with $\bar{\beta}^{0}=\mathcal{P}_{1} \hat{\beta}_{0}$ and $\eta_{i}=\mathcal{P}_{1} e_{i}$. Then $\mathcal{N}=\mathcal{P}_{1} \mathbb{R}^{m}$ is a parallel flat normal subbundle $\mathcal{N}$ of rank $m$ of $h=\mathcal{R}_{\varphi_{1}, \beta_{1}, \Omega_{11}}(g)$ and

$$
G(x, t)=\mathcal{R}_{\bar{\varphi}_{0}, \bar{\beta}_{0}+t}(h)(x)=\mathcal{R}_{\bar{\varphi}_{0}, \bar{\beta}_{0}}^{\mathcal{N}}(h)(t),
$$

where $t=\sum_{i=1}^{m} t_{i} \eta_{i}$. Moreover, the assumption on the bilinear map $\gamma$ is easily seen to be equivalent to the subspaces

$$
E(x)=\left\{Z \in T_{x} L:\left(\alpha_{h}(Z, X)\right)_{\mathcal{N}^{\perp}}=-\bar{\varphi}_{0}^{-1} \bar{\beta}_{\mathcal{N}^{\perp}}\langle Z, X\rangle \text { for all } X \in T_{x} L\right\}
$$

being everywhere trivial. By the result of [6] discussed before the statement of Theorem 22, it follows that $G$ parameterizes, at regular points, an $n$-dimensional submanifold carrying a Dupin principal normal of multiplicity $m$ with integrable conullity.

Conversely, given a submanifold $f: M^{m} \rightarrow \mathbb{R}^{N}$ that carries a Dupin principal normal of multiplicity $m$ with integrable conullity, by the aforementioned result of [6] there exist an isometric immersion $h: L^{n-m} \rightarrow \mathbb{R}^{N}$ carrying a parallel flat normal subbundle $\mathcal{N}$ of rank $m, \varphi \in C^{\infty}\left(L^{n-m}\right)$ and $\beta \in \Gamma\left(T_{h}^{\perp} L\right)$ satisfying $\alpha(X, \nabla \varphi)+\nabla \frac{\perp}{X} \beta=0$, with

$$
E(x)=\left\{Z \in T_{x} L:(\alpha(Z, X))_{\mathcal{N}^{\perp}}=-\varphi^{-1} \beta_{\mathcal{N}^{\perp}}\langle Z, X\rangle \text { for all } X \in T_{x} L\right\}
$$

everywhere trivial, such that $f$ is parameterized by the map $\mathcal{R}_{\varphi, \beta}^{\mathcal{N}}(h): L^{n-m} \times$ $\mathbb{R}^{m} \rightarrow \mathbb{R}^{N}$ given by

$$
\mathcal{R}_{\varphi, \beta}^{\mathcal{N}}(h)(x, t)=\mathcal{R}_{\varphi, \beta+t^{\prime}}(h)(x)
$$

where $t^{\prime}=\sum_{i=1}^{m} t_{i} \eta_{i}$ for some orthonormal parallel frame $\eta_{1}, \ldots, \eta_{m}$ of $\mathcal{N}$.

As in the proof of Theorem 18 there is an isometric immersion $g: L^{n-m} \rightarrow$ $\mathbb{R}^{N-m} \subset \mathbb{R}^{N}$ such that $h=\mathcal{R}_{\varphi_{1}, \beta_{1}, \Omega_{11}}(g)$, and hence

$$
\mathcal{R}_{\varphi, \beta}^{\mathcal{N}}(h)(x, t)=\mathcal{R}_{\varphi, \beta+t^{\prime}}\left(\mathcal{R}_{\varphi_{1}, \beta_{1}, \Omega_{11}}(g)\right)(x) .
$$

In order to apply Corollary 17, we must verify that the tensor $\Phi=\operatorname{Hess} \varphi-A_{\beta}$ associated to $(\varphi, \beta)$ commutes with $\tilde{\Phi}_{v_{1}}$ for every $v_{1} \in V_{1}$, where $D_{1} \bar{\Phi}_{v_{1}}^{1}=-\Phi_{\Omega_{11}^{-1} v_{1}}^{1}$ for $\Phi^{1}=\Phi\left(d \varphi_{1}, \beta_{1}\right)$. Since $\Phi$ commutes with the shape operator $\tilde{A}_{\tilde{\xi}}$ of $h$ with respect to any normal vector field $\tilde{\xi} \in \Gamma\left(T_{h}^{\perp} L\right)$, it commutes in particular with 
$\tilde{A}_{\mathcal{P}_{1} e_{i}}$. But by (21) we have

$$
\tilde{A}_{\mathcal{P}_{1} e_{i}}=D_{1}^{-1} \Phi_{\Omega_{11}^{-1} \beta_{1}^{t} e_{i}}^{1}=D_{1}^{-1} \Phi_{\Omega_{11}^{-1} e_{i}}^{1}=-\bar{\Phi}_{e_{i}}^{1},
$$

and we are done. It follows from Corollary 17 that there exist $(\varphi, \beta, \Omega)$ satisfying the conditions of Definition 9 with respect to $g$ and an orthogonal decomposition $\mathbb{R}^{m+1}=\mathbb{R} \oplus \mathbb{R}^{m}$ such that

$$
\left(\varphi_{1}, \beta_{1}, \Omega_{11}\right)=\left(\pi_{\mathbb{R}^{m}} \circ \varphi,\left.\beta\right|_{\mathbb{R}^{m}},\left.\pi_{\mathbb{R}^{m}} \circ \Omega\right|_{\mathbb{R}^{m}}\right)
$$

and, setting $\varphi_{0}=\pi_{\mathbb{R}} \circ \varphi, \beta_{1}=\left.\beta\right|_{\mathbb{R}}$, then $(\varphi, \beta)=\mathcal{R}_{\varphi, \beta, \Omega}\left(\varphi_{0}, \beta_{0}\right)$ and

$$
\mathcal{R}_{\varphi, \beta}\left(\mathcal{R}_{\varphi_{1}, \beta_{1}, \Omega_{11}}(g)\right)=\mathcal{R}_{\varphi, \beta, \Omega}(g) .
$$

Defining $\beta_{t}$ and $\Omega_{t}$ as in the statement, we have

$$
\mathcal{R}_{\varphi, \beta+t^{\prime}}\left(\mathcal{R}_{\varphi_{1}, \beta_{1}, \Omega_{11}}(g)\right)=\mathcal{R}_{\varphi, \beta_{t}, \Omega_{t}}(g) .
$$

\section{ACKNOWLEDGMENT}

We are grateful to the referee for useful remarks and for bringing to our attention the papers [9] and [10. As pointed out by him, in those articles a canonical correspondence between Hamiltonian systems of hydrodynamic type and hypersurfaces of a (pseudo) Euclidean space is provided, and that construction coincides when $m=1$ with (in fact it is the "Legendre dual" of) the one given by Theorem 1. He also remarks that commuting families of Hessian matrices arise in the theory of Hamiltonian systems of hydrodynamic type by Dubrovin, Novikov and Tsarev among others.

\section{REFERENCES}

[1] J. Berndt, S. Console and C. Olmos, Submanifolds and Holonomy, CRC/Chapman and Hall Research Notes Series in Mathematics 434 (2003), Boca Raton. MR1990032(2004e:53073)

[2] F.E. Burstall and U. Hertrich-Jeromin, The Ribaucour transformation in Lie sphere geometry, Preprint arXiv:math.DG/0407244 v1, 2004.

[3] G. Darboux, Leçons sur les systèmes orthogonaux et les coordonnées curvilignes, GauthierVillars, Paris, 1910.

[4] M. Dajczer and R. Tojeiro, An extension of the classical Ribaucour transformation. Proc. London Math. Soc. 85 (2002), 211-232. MR1901374 (2003g:53091)

[5] M. Dajczer and R. Tojeiro, Commuting Codazzi tensors and the Ribaucour transformations for submanifolds. Result. Math. 44 (2003), 258-278. MR2028680 (2005g:53027)

[6] M. Dajczer, L. Florit and R. Tojeiro, Reducibility of Dupin submanifolds. Illinois J. Math. 49 (2005), 759-791. MR2210258

[7] D. Ferus, A remark on Codazzi tensors in constant curvature spaces. Lect. Notes in Math. 838, Berlin 1981, 247. MR0636289 (83e:53045)

[8] E. V. Ferapontov, Surfaces with flat normal bundle: an explicit construction. Diff. Geom. Appl. 14 (2001), 15-37. MR1811562 (2002c:53006)

[9] E. V. Ferapontov, Hamiltonian systems of hydrodynamic type and their realization on hypersurfaces of a pseudoeuclidean space. Soviet J. of Math. 55 (1991), 1970-1995. MR1099220 (92f:58159)

[10] E. V. Ferapontov, Dupin hypersurfaces and integrable Hamiltonian systems of hydrodynamic type, which do not possess Riemann invariants. Diff. Geom. Appl. 5 (1995), 121-152. MR.1334839 (96d:53071)

[11] E. Ghanza and S. Tsarev, An algebraic superposition formula and the completeness of Bäcklund transformations of $(2+1)$-dimensional integrable systems. Uspekhi Mat. Nauk $\mathbf{5 1}$ (1996), 197-198; translation in Russian Math. Surveys 51 (1996), 1200-1202. MR1440161 (98b:58153) 
[12] U. Hertrich-Jeromin, Introduction to Möbius differential geometry, London Mathematical Society Lecture Note Series, vol. 300, Cambridge University Press, Cambridge, 2003. MR.2004958

[13] Q. P. Liu and M. Manas, Vectorial Ribaucour transformation for the Lamé equations. J. Phys. A. 31 (1998), 193-200. MR.1629398 (99c:58178)

impa, Estrada Dona Castroina, 110, 22460-320, Rio de Janeiro, BraziL

E-mail address: marcos@impa.br

impa, Estrada Dona Castroina, 110, 22460-320, Rio de Janeiro, Brazil

E-mail address: luis@impa.br

Universidade Federal de São Carlos, Via Washington Luiz km 235, 13565-905, São Carlos, Brazil

E-mail address: tojeiro@dm.ufscar.br 